\title{
APPLICATION OF THE DISCONTINUOUS GALERKIN TIME DOMAIN METHOD TO THE SIMULATION OF THE OPTICAL PROPERTIES OF DIELECTRIC PARTICLES
}

\author{
A Thesis \\ by \\ GUANGLIN TANG
}

\begin{abstract}
Submitted to the Office of Graduate Studies of Texas A\&M University

in partial fulfillment of the requirements of the degree of

MASTER OF SCIENCE
\end{abstract}

May 2010

Major Subject: Atmospheric Sciences 


\title{
APPLICATION OF THE DISCONTINUOUS GALERKIN TIME DOMAIN METHOD TO THE SIMULATION OF THE OPTICAL PROPERTIES OF DIELECTRIC PARTICLES
}

\author{
A Thesis \\ by \\ GUANGLIN TANG
}

\begin{abstract}
Submitted to the Office of Graduate Studies of Texas A\&M University in partial fulfillment of the requirements of the degree of

MASTER OF SCIENCE
\end{abstract}

Approved by:

Co-Chairs of Committee, $\quad$ R. Lee Panetta Ping Yang George W. Kattawar

Committee Members, Kenneth Bowman

May 2010

Major Subject: Atmospheric Sciences 


\begin{abstract}
Application of the Discontinuous Galerkin Time Domain

Method to the Simulation of the Optical Properties of

Dielectric Particles. (May 2010)

Guanglin Tang, B.S., Peking University

Co-Chairs of Advisory Committee: Dr. R. Lee Panetta

Dr. Ping Yang
\end{abstract}

A Discontinuous Galerkin Time Domain method (DGTD), using a fourth order Runge-Kutta time-stepping of Maxwell's equations, was applied to the simulation of the optical properties of dielectric particles in two-dimensional (2-D) geometry. As examples of the numerical implementation of this method, the single-scattering properties of $2 \mathrm{D}$ circular and hexagonal particles are presented. In the case of circular particles, the scattering phase matrix was computed using the DGTD method and compared with the exact solution. For hexagonal particles, the DGTD method was used to compute singlescattering properties of randomly oriented 2-D hexagonal ice crystals, and results were compared with those calculated using a geometric optics method.

Both shortwave (visible) and longwave (infrared) cases are considered, with particle size parameters 50 and 100. Ice in shortwave and longwave cases is absorptive and non-absorptive, respectively. The comparisons between DG solutions and the exact solutions in computing the optical properties of circular ice crystals reveal the applicability of the DG method to calculations of both absorptive and non-absorptive particles. In the hexagonal case scattering results are also presented as a function of both 
incident and scattering angles, revealing structure apparently not reported before. Using the geometric optics method we are able to interpret this structure in terms of contributions from varying numbers of internal reflections within the crystal. 


\section{ACKNOWLEDGEMENTS}

This study is partly supported by the National Science Foundation (NSF) under grant ATM-0803779.

I thank Tim Warburton for providing the original DGTD code and for useful guidance. Most of the computational work was done using resources of the Texas A\&M Supercomputer Facility. I thank Xiandong Meng and Ping Luo at this Facility for their help in porting the code.

I would like to express my appreciation to my advisors, Dr. Lee Panetta and Dr. Ping Yang, for their advising in my research and course work. Without their continuous help and encouragement, I could not think of finishing my master study in less than 2 years. The detailed appreciation will be too long for this thesis.

I also thank my committee member, Dr. George Kattawar. He is an expert in radiation transfer and has given me a lot of valuable suggestions for my research.

I thank my group members, Yu Xie, Yue Li, Lei Bi, Bingqi Yi, Qian Feng, Shouguo Ding for their help and advice in my study and life. I learned many usefull computer techniques from Mr. Yi. I thank Zhaokai Meng for discussing academics with me. He is good at finding new methods and I learned a lot from him. Thank you Kai Lu, Chenxi Wang and Chao Liu for sharing fun times with me.

Great appreciation goes to my family for their endless love. 
TABLE OF CONTENTS

Page

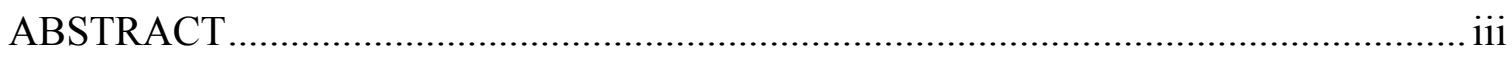

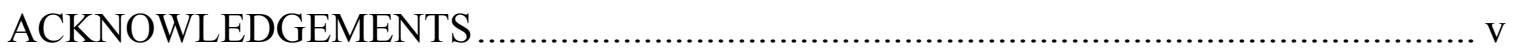

TABLE OF CONTENTS ............................................................................... vi

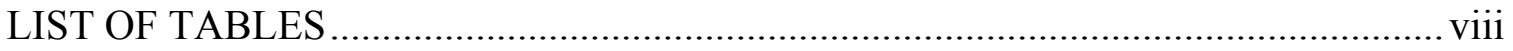

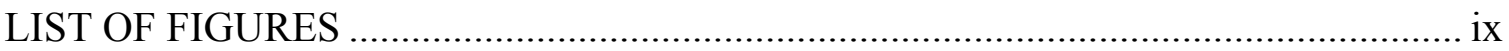

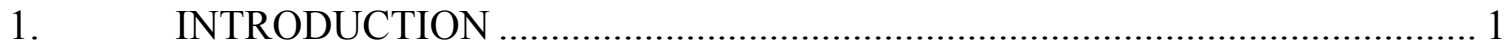

2. PHYSICAL BACKGROUND .............................................................. 6

2.1 The amplitude scattering matrix and the scattering phase matrix............. 6

2.2 Maxwell's equations in dielectric media ......................................... 10

3. DISCONTINUOUS GALERKIN TIME DOMAIN METHOD FOR COMPUTATION OF OPTICAL PROPERTIES OF DIELECTRIC

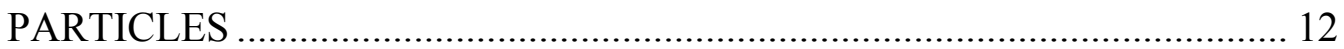

3.1 Governing equations .............................................................. 12

3.2 Boundary conditions and the unixial perfect matched layer.................... 14

3.3 Discontinuous Galerkin formulations .............................................. 16

3.4 The transformation of the near field to the far field................................ 22

4. EXTENSION OF THE DISCONTINUOUS GALERKIN METHOD TO 3-DIMENSIONAL CALCULATIONS OF SINGLE PARTICLE OPTICAL PROPERTIES ...................................................................... 25

4.1 Semi-discrete form of the governing equations for the near field

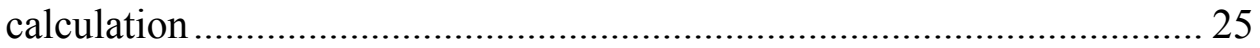

4.2 Transformation of the near field to the far field ................................. 28

5. NUMERICAL RESULTS AND DISCUSSIONS ................................... 30

5.1 Investigation of the applicability of the Discontinuous Galerkin Time-Domain method to the computation of light scattering properties of circular particles .......................................................... 30

5.2 Application of the Discontinuous Galerkin Time-Domain method to the computation of light scattering properties of hexagonal ice crystal. 
5.3 Applicability of the Discontinuous Galerkin Time-Domain method to scattering problems in 3-dimensional geometry ................... 36

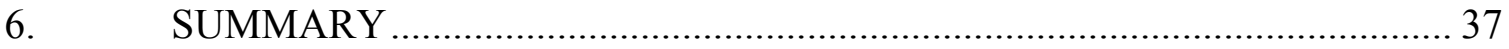

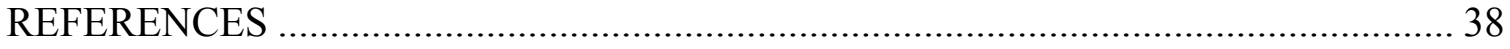

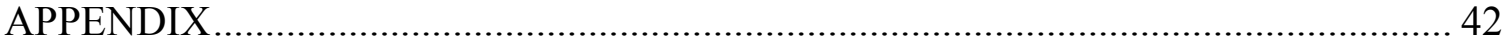

VITA

56 


\section{LIST OF TABLES}

TABLE

Page

1. Set of DGTD simulations and the computer time consumed. All calculations done on an IBM p5-575+ cluster, and only the first time reported is in hours. The remaining times are recorded as multiples of this time. 


\section{LIST OF FIGURES}

FIGURE

1 Geometry of light scattering process of a particle

2 Left: Grid for the simulation of an infinitely long circular cylinder. Right: Grid for the simulation of a hexagon. The size parameters are both 10. (Note: the sizes are expressed in dimensional units.) .....

3 Left Panel: Nonzero phase matrix elements of an infinitely long circular cylinder with a size parameter of 50 and an incident wavelength of $0.532 \mu \mathrm{m}$.

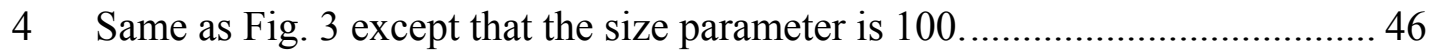

5 The same as Fig. 3 except that the incident wavelength is $12 \mu \mathrm{m} \ldots \ldots \ldots \ldots \ldots \ldots . . . . . . . .47$

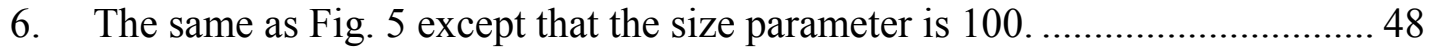

$7 \quad$ Nonzero scattering phase matrix elements of randomly oriented 2-D hexagonal ice crystals with a size parameter of 50 and an incident wavelength of $0.532 \mu \mathrm{m}$.

8 The same as Fig. 6 except that the size parameter is 100 .

9 Contributions of the rays of various orders to the phase function of 2D randomly oriented hexagonal ice crystals.

10 Contour of the $\log$ of the phase function with respect to scattering angle and incident angle, for hexagonal particles with a size parameter of 100 and an incident wave length of $0.532 \mu \mathrm{m}$, where the incident angle $\left(\theta_{\mathrm{i}}\right)$ and scattering angle $\left(\theta_{\mathrm{i}}\right)$ are defined in the lower panel.

11 Decomposition of the scattered field using geometrical optics. 53

12 An example of tetrahedron grid used in simulations of optical properties of a spherical particle. The green tetrahedrons make up the spherical particle, the red ones make up the "free space" and the blue ones make up the UPML

13 The $\mathrm{x}$ component of the electric field at a point of the free space with respect to time. 


\section{INTRODUCTION}

The atmospheric particles include mainly air molecular, aerosol, ice crystals and rain droplets. These atmospheric particles scatter and absorb more than half of the incoming solar radiation and the outgoing earth radiation. Cirrus clouds, which compose mainly of ice crystals and which cover more than $20 \%$ of the global surface, play important roles in the earth energy balance and in the research of temperature retrieval from satellite data. The aerosol, in a number of various types, e.g., dust, soot and clusters of them, which are responsible for the cloud formation, are present in the atmosphere nearly everywhere, and their optical properties are being studied by many researchers because of their big effect on the retrievals. The scattering properties of air molecular can be computed using the Rayleigh scattering theory, while those of the droplets, as spherical particles, can be computed using the Mie theory. For aerosol and ice crystals with large sizes (with size parameter of larger than 20), one can use a geometric optics method, which is an approximate method, but for those with moderate size (with size parameter between 1 20) and non-spherical geometries one must use numerical methods.

Numerous analytic and numerical methods have been developed for the simulation of the optical properties of particles with nonspherical geometries [1-4], including the T-matrix method [5,6], the Finite Difference Time Domain (FDTD) technique [7-10], the Pseudo-Spectral Time Domain (PSTD) method [11-13], and the Discrete Dipole Approximation (DDA) [14-16]. During the last decade discontinuous Galerkin (DG) methods, with versions in both the frequency domain (DGFD) and the

This thesis follows the style of Applied Optics. 
time domain (DGTD), have been developed and show considerable promise for the simulation of electromagnetic wave propagation, particularly in scattering simulations involving bodies of complex shapes. DG methods may be considered to be a hybrid of finite element, finite volume, and spectral methods, sharing valuable features of each. Frequency domain DG methods were introduced in the study of Maxwell's equations by Perugia and Schötzau [17] (see also Houston et. al. [18]). Time domain DG methods were introduced by Hesthaven and Warburton [19]. More information on the early history of DG methods can be found in Cockburn et. al. [20], and a historical sketch including discussion of more recent refinement of DG methods in the study of Maxwell's equations can be found in [21].

Similar to finite volume and finite element methods, discontinuous Galerkin methods typically use unstructured grids that can well approximate boundaries having geometric complexity, thereby avoiding the "stair-stepping" effects that occur with simple structured grids used in finite difference methods, effects that can result in numerical dispersion and dissipation that may significantly degrade the simulations (Cangellaris \& Wright [22]). At the same time, DG methods share with spectral methods the ability use a relatively coarse spatial mesh by using polynomials of arbitrarily high order on individual elements of the mesh. DG methods may be regarded as "localized Galerkin" methods, in the sense that solutions on individual elements are expressed as linear combinations of basic funsctions, in this case polynomial functions. In the version we use the linear combination represents an interpolation of solution values at specified points ("nodes") within the element and on its boundary, using Lagrange polynomials of 
user-chosen order "p". In general, therefore, DG methods offer two kinds of refinement: mesh refinement (or "h-refinement") and polynomial order refinement (“p-refinement").

In comparison with global spectral methods, which have difficulties handling either complex geometry or discontinuities in media, the local DG representations allow much better treatment of geometry and discontinuities. Approximations are permitted that are discontinuous at boundaries (hence the name of the method): the approximations on adjacent elements are connected not by continuity requirements at shared boundaries, but instead by flux conditions imposed at those boundaries. As in finite difference methods, choices are available that implement "upwind," "centered," or "downwind" fluxes, and the implementation is through specification of what are called "numerical fluxes." An excellent discussion of the theory and implementation may be found in [21].

The computed results given in the thesis concerning scattering from single dielectric particles using a version of the DGTD method adapted from that presented in [21]. The low-storage fourth-order, five-stage Runge-Kutta method (LSERK) [23] is used in the time discretization. An anisotropic Uniaxial Perfectly Matched Layer (UPML) absorbing boundary condition [24] is applied to suppress artificial reflections from the computational boundary. The DG method is used to calculate near-field scattering, and a surface-integral-based mapping scheme was used to calculate the far field from the near field [30].

Our interest is in the computationally difficult regime of large particle size (as measured in wavelengths of incident radiation) and asymmetric geometry. We are currently extending our DGTD method to three-dimensional simulations, but here just consider two simpler 2-dimensional scattering problems that give some indication of the 
power of the DGTD method. In each case the problem may be considered that of the scattering produced by an infinitely long cylinder (or very long cylinder with neglect of end effects), when the direction of propagation of the incident wave is normal to the axis of the cylinder. In the first case we consider circular geometry, for which an exact solution is known [25], and our DGTD results will be shown to give an excellent reproduction of the exact solution. In the second case, we consider hexagonal geometry, for which exact results are not available, and we instead compare the DGTD solution under random orientation conditions with the approximation obtained using the Improved Geometric Optics Method (IGOM) [30]. The DGTD and IGOM methods produce results that are also in a close agreement.

The "random" orientation calculation assumes equal probability of all particle orientations, and is simply an average of the scattering properties over the incident angles. We also present results in the case of hexagonal geometry without the averaging over orientation, and show the remarkable fine structure in the scattering field that appears not to have been noticed before. Using the IGOM method we demonstrate that much of this structure can be accounted for by considering rays with just a few internal reflections, the number of reflections being determined by the amount of absorption occurring within the particle.

In section 2 and 3 we describe the method. After a presentation of the TE and TM versions of the equations, we describe the particular PML scheme used. We then explain the representation of local solutions in terms of an interpolative expansion in Lagrange polynomials, and the manner in which the expansion leads to an evolution equation for "mass" and "stiffness" matrices. This explanation involves integration-by-parts 
arguments that introduce boundary integrals: the boundary integrals contain the numerical flux terms that tie the individual cell solutions together. The section concludes with a description of the near-to-far field solution transformation that allows approximation of the far field scattering field that is the quantity of interest.

Section 4 presents our results, in which we examine the scattered field for two different particle sizes (size parameters 50 and 100), and two different incident wavelengths. The size parameter is defined as $\frac{2 \pi a}{\lambda}$, where for a circle $a$ is the radius, and for the hexagon $a$ is the length of a side. The two wavelengths are chosen to correspond to the spectral peaks in incoming (shortwave) solar radiation and in outgoing (longwave) terrestrial radiation. 


\section{PHYSICAL BACKGROUND}

The scattering problems of individual particles are illustrated in theory in this section. The theoretical background includes the physical processes of the scattering problems, based on which, together with numerical schemes, the scattering problems are solved.

The light scattering of particles is a physical process where the light radiation changes its direction of propagation due to the existence of the particles. We focus on the scattering of single particles, partly because particles in the atmosphere are mostly sufficiently far from each other so that the scattering by one is not affected by the presence of others (except in some heavy clouds where multiple scattering of particles must be considered), and partly because the study of single particle scattering is the first step in the study of multiple scattering problems.

\subsection{The amplitude scattering matrix and the scattering phase matrix}

As a test of the DG method, the 2-dimensional case is considered. It corresponds to the situation of an infinitely long cylindrical particle with incident direction perpendicular to its axis. Fig. 1 illustrates the scattering process of a 2-dimensional particle: the 2-D particle is located in the X-Y plane. The directions of incident light and scattered light beams are also in $\mathrm{X}-\mathrm{Y}$ plane. Thus the $\mathrm{X}-\mathrm{Y}$ plane is always the principal scattering plane, defined as the plane which contains both the incident and scattered beams. One of components of the scattered far field $E_{/ /}^{s}$ is in the scattering plane and is 
perpendicular to the scattered beam, and the other component $E_{\perp}^{s}$ is perpendicular to the scattering plane and to the scattered direction. Likewise for the incident beam, the electric field is also decomposed into $E_{/ /}^{i}$ which is in the scattering plane and $E_{\perp}^{i}$ which is perpendicular to the scattering plane. In frequency domain, the electric far field and the incident field satisfy a relationship:

$$
\left(\begin{array}{l}
E_{/ /}^{s} \\
E_{\perp}^{s}
\end{array}\right)=\frac{e^{i k(r-x)}}{-i k r}\left(\begin{array}{ll}
S_{2} & S_{3} \\
S_{4} & S_{1}
\end{array}\right)\left(\begin{array}{l}
E_{/ /}^{s} \\
E_{\perp}^{s}
\end{array}\right)
$$

where $\mathrm{S} 1, \mathrm{~S} 2, \mathrm{~S} 3$ and $\mathrm{S} 4$ are the elements of the amplitude scattering matrix $\mathrm{S}, \mathrm{r}$ is the distance from the particle, and $\mathrm{k}$ is the wave number of the incident field. The scattering matrix $\mathrm{S}$ is a function of the scattering angle $\theta$. The function depends on the particle shape, size and orientation.

The amplitude scattering matrix S represents the scattering properties of a particle in a chosen orientation. However, to measure the amplitude scattering matrix, one needs to measure both the amplitude and phase of the incident and scattering field. A difficulty is met when measuring the phase of the field. Another difficulty emerges when computing the scattering properties of a random oriented particle. Thus for ease of measurement, an irradiant representation of the scattering properties is derived:

$$
\left(\begin{array}{l}
I^{s} \\
Q^{s} \\
U^{s} \\
V^{s}
\end{array}\right)=\frac{1}{k^{2} r^{2}}\left(\begin{array}{llll}
P_{11} & P_{12} & P_{13} & P_{14} \\
P_{21} & P_{22} & P_{23} & P_{24} \\
P_{31} & P_{32} & P_{33} & P_{34} \\
P_{41} & P_{42} & P_{43} & P_{44}
\end{array}\right)\left(\begin{array}{c}
I^{i} \\
Q^{i} \\
U^{i} \\
V^{i}
\end{array}\right)
$$

where $P_{i j}(i, j=1,2,3,4)$ are the elements of the scattering matrix. I, Q, U and V are Stokes parameters, which are practically measurable: 


$$
\begin{aligned}
& I=\left\langle E_{/ /} E_{/ /}^{*}+E_{\perp} E_{\perp}^{*}\right\rangle \\
& Q=\left\langle E_{/ /} E_{/ /}^{*}-E_{\perp} E_{\perp}^{*}\right\rangle \\
& U=\left\langle E_{/ /} E_{\perp}^{*}+E_{\perp} E_{/ /}^{*}\right\rangle \\
& V=\left\langle i\left(E_{/ /} E_{\perp}^{*}-E_{\perp} E_{/ /}^{*}\right)\right\rangle
\end{aligned}
$$

where the angular bracket \langle\rangle denotes the time average over a period of time that is much longer than the typical period of the quasi-monochromatic light beam. The vector $(I, Q, U, V) T$ is the Stokes vector. $I$ is the irradiance of the light. $Q, U$ and $V$ represent the polarization of the light. The Stokes vector of a light beam contains all of its information. If the incident light is direct solar radiation, for which $Q=U=V=0$, then $I^{s}=P_{11} * I^{i}, P_{11}$ which is called the phase function, represents the angular distribution of the scattering irradiance. Derived from the amplitude scattering matrix relationship and the definitions of the Stokes parameters, the scattering phase matrix can be expressed as: 


$$
\begin{aligned}
& P_{11}=\left(S_{1} S_{1}^{*}+S_{2} S_{2}^{*}+S_{3} S_{3}^{*}+S_{4} S_{4}^{*}\right) / 2 \\
& P_{12}=\left(-S_{1} S_{1}^{*}+S_{2} S_{2}^{*}-S_{3} S_{3}^{*}+S_{4} S_{4}^{*}\right) / 2 \\
& P_{13}=\operatorname{Re}\left(S_{1} S_{4}^{*}+S_{2} S_{3}^{*}\right) \\
& P_{14}=\operatorname{Im}\left(-S_{1} S_{4}^{*}+S_{2} S_{3}^{*}\right) \\
& P_{21}=\left(-S_{1} S_{1}^{*}+S_{2} S_{2}^{*}+S_{3} S_{3}^{*}-S_{4} S_{4}^{*}\right) / 2 \\
& P_{22}=\left(S_{1} S_{1}^{*}+S_{2} S_{2}^{*}-S_{3} S_{3}^{*}-S_{4} S_{4}^{*}\right) / 2 \\
& P_{23}=\operatorname{Re}\left(-S_{1} S_{4}^{*}+S_{2} S_{3}^{*}\right) \\
& P_{24}=\operatorname{Im}\left(S_{1} S_{4}^{*}+S_{2} S_{3}^{*}\right) \\
& P_{31}=\operatorname{Re}\left(S_{1} S_{3}^{*}+S_{2} S_{4}^{*}\right) \\
& P_{32}=\operatorname{Re}\left(-S_{1} S_{3}^{*}+S_{2} S_{4}^{*}\right) \\
& P_{33}=\operatorname{Re}\left(S_{1} S_{2}^{*}+S_{3} S_{4}^{*}\right) \\
& P_{34}=\operatorname{Im}\left(S_{1} S_{2}^{*}+S_{3} S_{4}^{*}\right) \\
& P_{41}=\operatorname{Im}\left(S_{1} S_{3}^{*}+S_{2} S_{4}^{*}\right) \\
& P_{42}=\operatorname{Im}\left(-S_{1} S_{3}^{*}+S_{2} S_{4}^{*}\right) \\
& P_{43}=\operatorname{Im}\left(S_{1} S_{2}^{*}-S_{3} S_{4}^{*}\right) \\
& P_{44}=\operatorname{Re}\left(S_{1} S_{2}^{*}-S_{3} S_{4}^{*}\right)
\end{aligned}
$$

Compared with the amplitude scattering matrix, the scattering phase matrix has advantages in representing the scattering properties. Beside the of ease measurement, it also has advantages in computing the scattering properties of randomly oriented particles using the results of various orientations. The scattering phase matrix of a randomly oriented particle is simply an average of the phase matrix over all incident angles.

To compute the amplitude scattering matrix, we need to compute the scattering fields in two modes, Transverse Magnetic or TM mode, and Transverse Electric or TE 
mode. In the TM mode, the electric field is always perpendicular to the scattering plane while the magnetic field is always in the scattering plane, or $E_{/ /}=0$. In the TE mode, the magnetic field is always perpendicular to the scattering plane while the electric field is always in the scattering plane, or $E_{\perp}=0$.

\subsection{Maxwell's equations in dielectric media}

Being an electromagnetic wave, light contains electric and magnetic fields which satisfy Maxwell's equations:

$$
\begin{aligned}
& \nabla \cdot \mathbf{D}=\rho_{f} \\
& \nabla \cdot \mathbf{B}=0 \\
& \nabla \times \mathbf{E}=-\frac{\partial \mathbf{B}}{\partial t} \\
& \nabla \times \mathbf{H}=\mathbf{J}_{f}+\frac{\partial \mathbf{D}}{\partial t}
\end{aligned}
$$

where $\mathbf{E}, \mathbf{B}, \mathbf{D}, \mathbf{H}, \mathbf{J}_{\mathrm{f}}$ and $\rho_{\mathrm{f}}$ are the electric field, the magnetic field, the electric displacement field, the magnetic field density, the free current density and the free charge density, respectively. For electromagnetic waves propagating in isotropic dielectric media, there are simple relationships between $\mathbf{E}$ and $\mathbf{D}$, between $\mathbf{B}$ and $\mathbf{H}$, and between $\mathbf{J}_{\mathrm{f}}$ and $\mathbf{E}$.

$$
\begin{aligned}
& \mathbf{D}=\varepsilon_{0} \varepsilon \mathbf{E} \\
& \mathbf{B}=\mu_{0} \mu \mathbf{H} \\
& \mathbf{J}_{f}=\sigma \mathbf{E}
\end{aligned}
$$

where $\varepsilon_{0}$ and $\mu_{0}$ are the permittivity and permeability of free space, $\varepsilon$ and $\mu$ are the relative permittivity and permeability of the media, and $\sigma$ is the conductivity of the media. 
Thus Maxwell's equations in isotropic dielectric media can be written as a set of two equations:

$$
\begin{aligned}
& \varepsilon_{0} \varepsilon \frac{\partial \mathbf{E}}{\partial t}=\nabla \times \mathbf{H}-\sigma \mathbf{E} \\
& \mu_{0} \mu \frac{\partial \mathbf{H}}{\partial t}=-\nabla \times \mathbf{E}
\end{aligned}
$$

For ease of introducing incident fields, the entire fields are decomposed into two parts: incident fields $\left(\mathbf{E}^{i}, \mathbf{H}^{i}\right)$ which satisfy Maxwell's equations in free space and scattered fields $\left(\mathbf{E}^{s}, \mathbf{H}^{s}\right)$ which are induced due to the existence of the scatterer:

$$
\begin{aligned}
& \mathbf{E}=\mathbf{E}^{i}+\mathbf{E}^{s} \\
& \mathbf{H}=\mathbf{H}^{i}+\mathbf{H}^{s}
\end{aligned}
$$

Substitution of eqs. (2.8) into eqs. (2.7) then gives:

$$
\begin{aligned}
& \varepsilon_{0} \frac{\partial \mathbf{E}^{i}}{\partial t}=\nabla \times \mathbf{H}^{i} \\
& \mu_{0} \frac{\partial \mathbf{H}^{i}}{\partial t}=-\nabla \times \mathbf{E}^{i} \\
& \varepsilon_{0} \varepsilon \frac{\partial \mathbf{E}^{s}}{\partial t}=\nabla \times \mathbf{H}^{s}+\varepsilon_{0}(1-\varepsilon) \frac{\partial \mathbf{E}^{i}}{\partial t}-\sigma\left(\mathbf{E}^{s}+\mathbf{E}^{i}\right) \\
& \mu_{0} \mu \frac{\partial \mathbf{H}^{s}}{\partial t}=-\nabla \times \mathbf{E}^{s}
\end{aligned}
$$

The scattering phase matrix can present the optical properties of atmospheric particles. Thus the main goal of solving scattering problems is to compute the scattering phase matrix. The governing equations in a decomposed form are solved in a time domain. Then the time domain fields are transformed into frequency domain fields using Fourier Transformation, and then be transformed into the far fields using a surfaceintegral scheme. Using both incident and scattering fields, the scattering phase matrix is calculated. 


\section{DISCONTINUOUS GALERKIN TIME DOMAIN METHOD FOR}

COMPUTATION OF OPTICAL PROPERTIES OF DIELECTRIC PARTICLES

\subsection{Governing equations}

For computational purposes, we non-dimensionalize Maxwell's equations in terms of four basic quantities: vacuum values of electric permittivity and magnetic permeability $\left(\varepsilon_{0}, \mu_{0}\right)$, unit magnetic field strength $H_{0}$, and wavelength of incident radiation $\lambda$. Using these we define non-dimensional variables $t=\lambda^{-1} c_{0} t^{*}, \quad(x, y, z)=\lambda^{-1}\left(x^{*}, y^{*}, z^{*}\right), \quad \mathbf{E}=\left(Z_{0} H_{0}\right)^{-1} \mathbf{E}^{*}, \quad \mathbf{H}=H_{0}^{-1} \mathbf{H}^{*}, \quad \sigma=\lambda Z_{0} \sigma^{*}$

where (here only) asterisks indicate dimensional quantities. The constants $c_{0}=\sqrt{\mu_{0}^{-1} \varepsilon_{0}^{-1}}$ and $Z_{0}=\sqrt{\mu_{0} \varepsilon_{0}^{-1}}$ are the vacuum values of the speed of light and impedance, respectively. Total field solutions are decomposed in the usual way into incident and scattered components, and then the general 3-D form of Maxwell's equations in a linear medium becomes (eg van de Hulst[26]):

$$
\begin{gathered}
\varepsilon \frac{\partial \mathbf{E}^{s}}{\partial t}=\nabla \times \mathbf{H}^{s}+(1-\varepsilon) \frac{\partial \mathbf{E}^{i}}{\partial t}-\sigma\left(\mathbf{E}^{s}+\mathbf{E}^{i}\right), \\
\mu \frac{\partial \mathbf{H}^{s}}{\partial t}=-\nabla \times \mathbf{E}^{s} .
\end{gathered}
$$

Here $\mathbf{E}$ and $\mathbf{H}$ denote the electric and magnetic fields; the superscripts $i$ and $s$ denote the incident and scattered fields, and $\varepsilon$ and $\sigma$ are the relative permittivity and the nondimensional conductivity [13] of the medium, respectively. Most particles in the atmosphere have a relative permeability of approximately 1, and in this study we assume 
$\mu$ to be 1 . The incident field vectors $\mathbf{E}^{i}$ and $\mathbf{H}^{i}$ are given, and the unknowns are the scattered field vectors $\mathbf{E}^{s}$ and $\mathbf{H}^{s}$.

In order to obtain the scattering properties, it is sufficient to consider the transverse magnetic (TM) and transverse electric (TE) cases. For the TM case, the magnetic field has non-zero components of incident and scattered waves only perpendicular to the symmetry axis of the cylinder, that is, in the plane of our crosssection, and the electric fields of both the incident and scattered waves have only nonzero components in the direction of the symmetry axis (perpendicular to that plane). For the TE case, it is the electric field that has non-zero components only in the plane perpendicular to the symmetry axis, and the magnetic field has its non-zero components parallel to that axis. The equations for the TM case are

$$
\begin{gathered}
\frac{\partial H_{x}^{s}}{\partial t}=-\frac{\partial E_{z}^{s}}{\partial y}, \\
\frac{\partial H_{y}^{s}}{\partial t}=\frac{\partial E_{z}^{s}}{\partial x}, \\
\varepsilon \frac{\partial E_{z}^{s}}{\partial t}=\frac{\partial H_{y}^{s}}{\partial x}-\frac{\partial H_{x}^{s}}{\partial y}+(1-\varepsilon) \frac{\partial E_{z}^{i}}{\partial t}-\sigma\left(E_{z}^{s}+E_{z}^{i}\right) .
\end{gathered}
$$

where the subscripts $\mathrm{x}, \mathrm{y}, \mathrm{z}$ denote the corresponding components of the field vectors, respectively.

For the TE case, the equations are:

$$
\begin{aligned}
& \varepsilon \frac{\partial E_{x}^{s}}{\partial t}=\frac{\partial H_{z}^{s}}{\partial y}+(1-\varepsilon) \frac{\partial E_{x}^{i}}{\partial t}-\sigma\left(E_{x}^{s}+E_{x}^{i}\right), \\
& \varepsilon \frac{\partial E_{y}^{s}}{\partial t}=-\frac{\partial H_{z}^{s}}{\partial x}+(1-\varepsilon) \frac{\partial E_{y}^{i}}{\partial t}-\sigma\left(E_{y}^{s}+E_{y}^{i}\right),
\end{aligned}
$$




$$
\frac{\partial H_{z}^{s}}{\partial t}=-\frac{\partial E_{y}^{s}}{\partial x}+\frac{\partial E_{x}^{s}}{\partial y}
$$

\subsection{Boundary conditions and the unixial perfect matched layer}

Our scattering problems involve an open boundary condition, which in computations must be implemented on a spatial domain of finite size. We divide the computational domain into three regions: the particle, a surrounding region of free space (relative permittivity 1), and an outer region in which is an anisotropic absorbing boundary layer. This layer prevents artificial reflection occurring at outer computational boundaries from re-entering the free space region at any appreciable amplitude (see below). The geometry is shown, using dimensional variables, in Fig. 2 for the two geometries used for our calculations. In the case of a particle with circular geometry, with dimensional radius $a$, and incident radiation of dimensional wavelength $\lambda$, the outer boundary of the free space region surrounding the particle is a square with sides of length $2 a+5 \lambda$, and the outer absorbing boundary layer has thickness another $5 \lambda$ in each horizontal direction. Thus the entire computational domain in this $2-\mathrm{d}$ problem is a square of dimensional length $2 a+15 \lambda$ on a side.

The Uniaxial Perfectly Matched Layer (UPML) absorbing boundary condition [24] is used in the outer layer. There, auxiliary variables $C_{x}^{s}, C_{y}^{s}$, and $D_{z}^{s}$, are defined, whose evolution couples with that of the scattered electromagnetic fields in a way that guarantees the stability of the UPML scheme [24].

The full set of equations in the UPML region in the TM case are

$$
\frac{\partial C_{x}^{s}}{\partial t}=-\frac{\partial E_{z}^{s}}{\partial y}-\sigma_{z} C_{x}^{s}
$$




$$
\begin{aligned}
& \frac{\partial C_{y}^{s}}{\partial t}=\frac{\partial E_{z}^{s}}{\partial x}-\sigma_{x} C_{y}^{s}, \\
& \frac{\partial D_{z}^{s}}{\partial t}=\frac{\partial H_{y}^{s}}{\partial x}-\frac{\partial H_{x}^{s}}{\partial y}-\sigma_{y} D_{z}^{s}, \\
& \frac{\partial H_{x}^{s}}{\partial t}=-\frac{\partial E_{z}^{s}}{\partial y}+\left(\sigma_{x}-\sigma_{z}\right) C_{x}^{s}-\sigma_{y} H_{x}^{s}, \\
& \frac{\partial H_{y}^{s}}{\partial t}=\frac{\partial E_{z}^{s}}{\partial x}+\left(\sigma_{y}-\sigma_{x}\right) C_{y}^{s}-\sigma_{z} H_{y}^{s}, \\
& \frac{\partial E_{z}^{s}}{\partial t}=\frac{\partial H_{y}^{s}}{\partial x}-\frac{\partial H_{x}^{s}}{\partial y}+\left(\sigma_{z}-\sigma_{y}\right) D_{z}^{s}-\sigma_{x} E_{z}^{s} .
\end{aligned}
$$

In (3.3a-3i) all variables are real: the auxiliary variables are initially set to zero. The quantities $\sigma_{x}, \sigma_{y}$ and $\sigma_{z}$ are functions of space that provide for the rapid (exponential [24]) decay of signals propagating in the PML layer, and are of the form

$$
\sigma_{s}=\sigma_{0}\left(\frac{s-s_{i}}{s_{o}-s_{i}}\right)^{m},
$$

where $s$ denotes $x, y$, or $z$; subscripts $i$ and $o$ denote the inner and outer boundaries of the PML region, respectively, and $m$ is an integer that controls the rate of decay. The constant $\sigma_{0}$ is

$$
\sigma_{0}=\frac{C(m+1)}{\left(s_{o}-s_{i}\right)} .
$$

with the choices $m=2$ and $C=15$, the amplitude of a scattered wave that has entered the PML, reflected at the computational boundary, and re-emerged from the PML will have diminished by at least a factor of $10^{-13}$. Note that the incident field is not affected by the PML region. 


\subsection{Discontinuous Galerkin formulations}

The grid for our computation is a tessellation of the computational domain in irregular triangle elements generated by the Persson and Strang MATLAB code [27]. In two dimensions, the Persson and Strang code provides the mesh by an iterative method, with desired length of an edge in the mesh as an input parameter: when given the input value of a half wavelength of the incident wave, the output is a mesh having a distribution of sizes between a half and a third of the wavelength. Examples are shown in Fig. 2 for crystals of both circular and hexagonal geometry. In the figure the size parameter is chosen to be 10 in order to make the grid structure visible (a total of $\sim 3700$ elements, with slight differences between the circular and hexagonal cases); our simulations used size parameters 50 and 100, giving meshes with approximately 11,000 and 27,000 elements, respectively (see Table 1). As mentioned above, the shortest distance between the boundary of the particle and the inner boundary of the PML layer is chosen to be $5 / 2$ times the incident wavelength, and the thickness of the PML layer is 5 times the wavelength.

The DG method we use calculates the values of field variables at a finite number of suitably chosen points ("nodes") in each element of the domain; values of field variables at other points are obtained by interpolation using Lagrange polynomials. Thus, within each element, field variables have the representation [17]:

$$
\begin{aligned}
& F(x, y, t)=\sum_{i=1}^{N_{p}} F\left(\xi_{i}, t\right) l_{i}(x, y), \\
& \frac{\partial F(x, y, t)}{\partial t}=\sum_{i=1}^{N_{p}} \frac{\partial F\left(\xi_{i}, t\right)}{\partial t} l_{i}(x, y),
\end{aligned}
$$




$$
\begin{aligned}
& \frac{\partial F(x, y, t)}{\partial x}=\sum_{i=1}^{N_{p}} F\left(\xi_{i}, t\right) \frac{\partial l_{i}(x, y)}{\partial x}, \\
& \frac{\partial F(x, y, t)}{\partial y}=\sum_{i=1}^{N_{p}} F\left(\xi_{i}, t\right) \frac{\partial l_{i}(x, y)}{\partial y},
\end{aligned}
$$

where $F(x, y, t)$ may be any function of space and time, for example an individual component of an electromagnetic field, or product of a component with a spatially varying field (eg. $\sigma_{z} E_{y}^{s}$ ). In such a representation, $F\left(\xi_{i}, t\right)$ is the value of $F$ at time $t$ at node $\xi_{i} ; l_{i}(x, y)$ is the $i^{\text {th }}$ Lagrange polynomial; and, $N_{p}$ is the number of nodes within each element. If the order of the polynomials is $N$, the number of nodes $N_{p}$ is:

$$
N_{p}=\frac{N(N+1)}{2}
$$

As mentioned in the introduction, one way to increase accuracy is to increase the $\operatorname{order} N$. This of course entails computational cost, and after some experimentation we settled on the choice $N=4$. The distribution of nodes in the element follows the $\alpha$ optimized nodal sets $[17,28]$.

In spectral methods, the central variables are the spectral amplitudes, and evolution equations for these amplitudes may be obtained by first substituting the spectral expansion into the field equations, then multiplying by individual spectral basis functions, and finally integrating over the (whole) domain. Terms involving derivatives of field variables lead through integration -by-parts arguments to boundary integrals that are evaluated using boundary conditions. A similar procedure is carried out here, but "localized" to inidividual elements: after substituting the expansion in Lagrange polynomials in the equations, each equation is multiplied by $l_{j}(x, y)$, where 
$j=1,2, \cdots, N_{p}$ and integrated over the area of the individual element. For terms that included a space derivative, the terms are integrated by parts and numerical flux terms are introduced. After integrating by parts again, we obtain the strong semi-discrete formulation for Eq. (3.3) (see [22]):

$$
\begin{array}{r}
\varepsilon \sum_{i=1}^{N_{p}} \frac{\partial \mathbf{E}^{s}\left(\xi_{i}, t\right)}{\partial t} \iint_{D_{k}} l_{i}(x, y) l_{j}(x, y) d x d y=-\sum_{i=1}^{N_{p}} \mathbf{H}^{s}\left(\xi_{i}, t\right) \times \iint_{D_{k}}\left[\nabla l_{i}(x, y)\right] l_{j}(x, y) d x d y \\
\frac{-\sum_{i=1}^{N_{p}}\left[\hat{\mathbf{n}} \times \mathbf{H}^{s}\left(\xi_{i}, t\right)-\left(\hat{\mathbf{n}} \times \mathbf{H}^{s}\left(\xi_{i}, t\right)\right)^{*}\right] \oint_{\partial D_{k}} l_{i}(x, y) l_{j}(x, y) d s}{N_{p}}\left(1-\varepsilon\left(\xi_{i}, t\right)\right) \frac{\partial \mathbf{E}^{i}\left(\xi_{i}, t\right)}{\partial t} \iint_{D_{k}} l_{i}(x, y) l_{j}(x, y) d x d y \\
\sum_{i=1}^{N_{p}} \frac{\partial \sum_{i=1}^{N_{p}}\left(\xi_{i}, t\right)}{\partial t} \iint_{D_{k}} l_{i}(x, y) l_{j}(x, y) d x d y=\sum_{i=1}^{N_{p}} \mathbf{E}^{s}\left(\xi_{i}, t\right) \times \iint_{D_{k}}\left[\nabla l_{i}(x, y)\right] l_{j}(x, y) d x d y \\
+\sum_{i=1}^{N_{p}}\left[\hat{\mathbf{n}} \times \mathbf{E}^{s}\left(\xi_{i}, t\right)\right) \iint_{D_{k}} l_{i}(x, y) l_{j}(x, y) d x d y \\
\hline
\end{array}
$$

where $\partial D_{k}$ denotes the $k$ th triangle element; $\xi_{i}$ is the $i$ th node in the element; and the term $\left(\hat{n} \times \mathbf{H}^{s}\left(\xi_{i}, t\right)\right)^{*}$ indicates the numerical flux. An advantage of the nodal approach in the DG method is that, with some of the nodes located on the boundaries, the surface integrals (the underlined terms) may be calculated using only values at those nodes, without considering values at interior nodes. This means that the underlined terms in equations $(3.11 \mathrm{a}, 3.11 \mathrm{~b})$ can be rewritten as sums over a smaller number of surface nodes:

$$
\begin{aligned}
\sum_{i=1}^{N_{p}}\left[\hat{\mathbf{n}} \times \mathbf{H}^{s}\left(\xi_{i}, t\right)-\left(\hat{\mathbf{n}} \times \mathbf{H}^{s}\left(\xi_{i}, t\right)\right)^{*}\right] \oint_{\partial D_{k}} l_{i}(x, y) l_{j}(x, y) d s \\
=\sum_{s=1}^{3 N_{f p}}\left[\hat{\mathbf{n}} \times \mathbf{H}^{s}\left(\xi_{i_{s}}, t\right)-\left(\hat{\mathbf{n}} \times \mathbf{H}^{s}\left(\xi_{i_{s}}, t\right)\right)^{*}\right] \oint_{\partial D_{k}} l_{i_{s}}(x, y) l_{j}(x, y) d s
\end{aligned}
$$




$$
\begin{aligned}
\sum_{i=1}^{N_{p}}\left[\hat{\mathbf{n}} \times \mathbf{E}^{s}\left(\xi_{i}, t\right)-\left(\hat{\mathbf{n}} \times \mathbf{E}^{s}\left(\xi_{i}, t\right)\right)^{*}\right] \oint_{\partial D_{k}} l_{i}(x, y) l_{j}(x, y) d s \\
=\sum_{s=1}^{3 N_{f p}}\left[\hat{\mathbf{n}} \times \mathbf{E}^{s}\left(\xi_{i_{s}}, t\right)-\left(\hat{\mathbf{n}} \times \mathbf{E}^{s}\left(\xi_{i_{s}}, t\right)\right)^{*}\right] \oint_{\partial D_{k}} l_{i_{s}}(x, y) l_{j}(x, y) d s
\end{aligned}
$$

where $N_{f p}$ is the number of nodes on one face of a triangular element, $N_{f p}=N+1, \xi_{i_{s}}$ is the coordinate of the node on the surface, and $i_{s}$ is the index of nodes on the element surface. On each vertex of the trianglar element, the flux term, $\hat{\mathbf{n}} \times \mathbf{H}^{s}\left(\xi_{i_{s}}, t\right)-\left(\hat{\mathbf{n}} \times \mathbf{H}^{s}\left(\xi_{i_{s}}, t\right)\right)^{*}$, is counted twice, with $\hat{\mathbf{n}}$ corresponding to the two edges sharing the vertex.

To rewrite the equations in matrix form, the matrices below are defined:

$$
\begin{aligned}
& \mathbf{M}_{i j}=\iint_{D_{k}} l_{j}(x, y) l_{i}(x, y) d x d y, \\
& \mathbf{S}_{i j}^{x}=\iint_{D_{k}} \frac{\partial l_{j}(x, y)}{\partial x} l_{i}(x, y) d x d y, \\
& \mathbf{S}_{i j}^{y}=\iint_{D_{k}} \frac{\partial l_{j}(x, y)}{\partial y} l_{i}(x, y) d x d y, \\
& \mathbf{C}_{i s}=\oint_{\partial D_{k}} l_{j_{s}}(x, y) l_{i}(x, y) d s .
\end{aligned}
$$

$\mathbf{M}_{i j}, \mathbf{S}_{i j}^{x}, \mathbf{S}_{i j}^{y}$ are $N_{p} \times N_{p}$ matrices, with $i$ and $j$ denoting nodes in the element (including boundary nodes). $\mathbf{C}_{i s}$ is $N_{p} \times 3 N_{f p}$ matrix, where subscript $j_{s}$ denotes the index of a node on the element surface, and the vertices are each counted twice.

The numerical fluxes for Maxwell's equations are derived by applying the Rankine-Hugoniot conditions [29], and is expressed as follows [21]:

$$
\hat{\mathbf{n}} \times \mathbf{H}^{s}-\left(\hat{\mathbf{n}} \times \mathbf{H}^{s}\right)^{*}=\frac{1}{Z^{-}+Z^{+}} \hat{\mathbf{n}} \times\left[Z^{+}\left(\mathbf{H}^{s-}-\mathbf{H}^{s+}\right)-\alpha \hat{\mathbf{n}} \times\left(\mathbf{E}^{s-}-\mathbf{E}^{s+}\right)\right],
$$




$$
\hat{\mathbf{n}} \times \mathbf{E}^{s}-\left(\hat{\mathbf{n}} \times \mathbf{E}^{s}\right)^{*}=\frac{1}{Y^{-}+Y^{+}} \hat{\mathbf{n}} \times\left[Y^{+}\left(\mathbf{E}^{s-}-\mathbf{E}^{s+}\right)+\alpha \hat{\mathbf{n}} \times\left(\mathbf{H}^{s-}-\mathbf{H}^{s+}\right)\right],
$$

where $\alpha$ is a number between 0 and 1 . The numerical flux is a central flux when $\alpha=0$ and an upwind flux when $\alpha=1$. The upwind flux is what we use. $Z^{ \pm}$and $Y^{ \pm}$are functions of the material coefficients with the symbols "-" and "+" denoting the coefficients on the inner and outer surfaces of the element, respectively,

$$
Z^{ \pm}=\frac{1}{Y^{ \pm}}=\sqrt{\frac{\mu^{ \pm}}{\varepsilon^{ \pm}}}
$$

All of these matrices can be calculated. Subsequently, Eq. (3.11) can be written in the matrix form:

$$
\begin{gathered}
\frac{d \mathbf{E}^{s}}{d t}=\frac{1}{\varepsilon}\left[\mathbf{M}^{-1} \mathbf{S} \times \mathbf{H}^{s}+\mathbf{M}^{-1} \mathbf{C}\left(\hat{\mathbf{n}} \times \mathbf{H}^{s}-\left(\hat{\mathbf{n}} \times \mathbf{H}^{s}\right)^{*}\right)+(1-\varepsilon) \frac{\partial \mathbf{E}^{i}}{\partial t}-\sigma\left(\mathbf{E}^{i}+\mathbf{E}^{s}\right)\right], \\
\frac{d \mathbf{H}^{s}}{d t}=-\mathbf{M}^{-1} \mathbf{S} \times \mathbf{E}^{s}-\mathbf{M}^{-1} \mathbf{C}\left(\hat{\mathbf{n}} \times \mathbf{E}^{s}-\left(\hat{\mathbf{n}} \times \mathbf{E}^{s}\right)^{*}\right),
\end{gathered}
$$

where $\mathbf{H}^{s}$ and $\mathbf{E}^{s}$ are $\left(\mathrm{N}_{\mathrm{p}} \times 3\right)$ matrices whose (i,j) entry is the $\mathrm{j}^{\text {th }}$ component of the field at the $\mathrm{i}^{\text {th }}$ node

Substituting the numerical flux Eq. (3.17) into Eq. (3.19), and considering both the scattering region and the PML region, all the functions are rewritten in matrix form. In the particle and "free space" regions:

$$
\begin{aligned}
& \frac{\partial \widetilde{H}_{x}^{s}}{\partial t}=-\mathbf{M}^{-1} \mathbf{S}^{y} \widetilde{E}_{z}^{s}+\mathbf{M}^{-1} \mathbf{C} \widetilde{F}_{H x}^{s}, \\
& \frac{\partial \widetilde{H}_{y}^{s}}{\partial t}=\mathbf{M}^{-1} \mathbf{S}^{x} \widetilde{E}_{z}^{s}+\mathbf{M}^{-1} \mathbf{C} \widetilde{F}_{H y}^{s}, \\
& \varepsilon \frac{\partial \widetilde{E}_{z}^{s}}{\partial t}=\mathbf{M}^{-1} \mathbf{S}^{x} \widetilde{H}_{y}^{s}-\mathbf{M}^{-1} \mathbf{S}^{y} \widetilde{H}_{x}^{s}+\mathbf{M}^{-1} \mathbf{C} \widetilde{F}_{E z}^{s}+(1-\varepsilon) \frac{\partial \widetilde{E}_{z}^{i}}{\partial t}-\sigma\left(\widetilde{E}_{z}^{s}+\widetilde{E}_{z}^{i}\right) .
\end{aligned}
$$

In the PML region: 


$$
\begin{aligned}
& \frac{\partial \widetilde{C}_{x}^{s}}{\partial t}=-\mathbf{M}^{-1} \mathbf{S}^{y} \widetilde{E}_{z}^{s}+\mathbf{M}^{-1} \mathbf{C} \widetilde{F}_{H x}^{s}-\sigma_{z} \widetilde{C}_{x}^{s}, \\
& \frac{\partial \widetilde{C}_{y}^{s}}{\partial t}=\mathbf{M}^{-1} \mathbf{S}^{x} \widetilde{E}_{z}^{s}+\mathbf{M}^{-1} \mathbf{C} \widetilde{F}_{H y}^{s}-\sigma_{x} \widetilde{C}_{y}^{s}, \\
& \frac{\partial \widetilde{D}_{z}^{s}}{\partial t}=\mathbf{M}^{-1} \mathbf{S}^{x} \widetilde{H}_{y}^{s}-\mathbf{M}^{-1} \mathbf{S}^{y} \widetilde{H}_{x}^{s}+\mathbf{M}^{-1} \widetilde{C}_{E z}^{s}-\sigma_{y} \widetilde{D}_{z}^{s}, \\
& \frac{\partial \widetilde{H}_{x}^{s}}{\partial t}=-\mathbf{M}^{-1} \mathbf{S}^{y} \widetilde{E}_{z}^{s}+\mathbf{M}^{-1} \mathbf{C} \widetilde{F}_{H x}^{s}+\left(\sigma_{x}-\sigma_{z}\right) \widetilde{C}_{x}^{s}-\sigma_{y} \widetilde{H}_{x}^{s}, \\
& \frac{\partial \widetilde{H}_{y}^{s}}{\partial t}=\mathbf{M}^{-1} \mathbf{S}^{x} \widetilde{E}_{z}^{s}+\mathbf{M}^{-1} \mathbf{C} \widetilde{F}_{H y}^{s}+\left(\sigma_{y}-\sigma_{x}\right) \widetilde{C}_{y}^{s}-\sigma_{z} \widetilde{H}_{y}^{s}, \\
& \frac{\partial \widetilde{E}_{z}^{s}}{\partial t}=\mathbf{M}^{-1} \mathbf{S}^{x} \widetilde{H}_{y}^{s}-\mathbf{M}^{-1} \mathbf{S}^{y} \widetilde{H}_{x}^{s}+\mathbf{M}^{-1} \mathbf{C} \widetilde{F}_{E z}^{s}+\left(\sigma_{z}-\sigma_{y}\right) \widetilde{D}_{z}^{s}-\sigma_{x} \widetilde{E}_{z}^{s},
\end{aligned}
$$

where the numerical fluxes are derived from their vector forms (3.17):

$$
\begin{aligned}
& \widetilde{F}_{H x}^{s}=\frac{1}{Y^{-}+Y^{+}}\left\{\hat{n}_{y}\left(E_{z}^{s-}-E_{z}^{s+}\right) Y^{+}+\hat{n}_{x}\left[\hat{n}_{x}\left(H_{x}^{s-}-H_{x}^{s+}\right)+\hat{n}_{y}\left(H_{y}^{s-}-H_{y}^{s+}\right)\right]-\left(H_{x}^{s-}-H_{x}^{s+}\right)\right\} \\
& \widetilde{F}_{H y}^{s}=\frac{1}{Y^{-}+Y^{+}}\left\{-\hat{n}_{x}\left(E_{z}^{s-}-E_{z}^{s+}\right) Y^{+}+\hat{n}_{y}\left[\hat{n}_{x}\left(H_{x}^{s-}-H_{x}^{s+}\right)+\hat{n}_{y}\left(H_{y}^{s-}-H_{y}^{s+}\right)\right]-\left(H_{y}^{s-}-H_{y}^{s+}\right)\right\} \\
& \widetilde{F}_{E z}^{s}=\frac{1}{Z^{-}+Z^{+}}\left\{\left[-\hat{n}_{x}\left(H_{y}^{s-}-H_{y}^{s+}\right)+\hat{n}_{y}\left(H_{x}^{s-}-H_{x}^{s+}\right)\right] Z^{+}-\left(E_{z}^{s-}-E_{z}^{s+}\right)\right\}
\end{aligned}
$$

here $\widetilde{H}_{x}^{s}, \widetilde{H}_{y}^{s}, \widetilde{E}_{z}^{s}, \widetilde{C}_{x}^{s}, \widetilde{C}_{y}^{s}, \widetilde{D}_{z}^{s}$ are $N_{p} \times 1$ vectors, whose values are the corresponding field values on the nodal points which are denoted by the index number of these vectors. $\widetilde{F}_{H x}^{s}, \widetilde{F}_{H y}^{s}$ and $\widetilde{F}_{E z}^{s}$ are $3 N_{f p} \times 1$ vectors, whose values are corresponding numerical flux at the surface nodal points which are denoted by the index number of these vectors.

The matrix form of the driving function and the numerical fluxes in the TE case is obtained following the same algorithms as in the TM case from Eq. (3.2). 
In the time integration, the low-storage fourth-order, five-stage Runge-Kutta (RK) method [23] was applied. After some experimentation with third order schemes, we settled on the fourth order scheme: it has two orders of accuracy more than a leap-frog scheme, and performs well with a high order special finite element scheme. Calculations were initialized with all scattered field variables initially set to zero, and continued until the solution appeared to settle into a steady state. (Steadiness was tested by integrating $25-50 \%$ longer and comparing results.)

\subsection{The transformation of the near field to the far field}

A scattering problem concerns the relation between an incident field and the scattered fiend at locations "far" from the particle, i.e., at distance large compared with both particle size and incident wavelength. Typically in practical simulations, the near field is calculated in a truncated domain covered by a fine spatial mesh, and then the far field is calculated using a Green's function technique. Here, after computing the near field using the DGTD method, we transform the field to the frequency domain and use a surface-integral based algorithm to calculate the far field and the scattering phase function. Details of the algorithm are presented in [30]. As an example we express the scattered electric field in terms of the near field and the Green's function in the TM mode:

$$
E_{s}^{z}(\mathbf{r})=\oint \hat{\mathbf{n}} \cdot\left[E_{s}^{z}\left(\mathbf{r}^{\prime}\right) \nabla^{\prime} G\left(\mathbf{r}, \mathbf{r}^{\prime}\right)-G\left(\mathbf{r}, \mathbf{r}^{\prime}\right) \nabla^{\prime} E_{s}^{z}\left(\mathbf{r}^{\prime}\right)\right] d r^{\prime}
$$

where the integral is performed at the surface of the particle, or any close curve which encloses the particle. In our simulations this surface is chosen to be the interface between 
the "free space" and the PML region. The Green's function G(r,r') in 2-D geometry is given in terms of the zero-order Hankel function of the first kind:

$$
G\left(\mathbf{r}, \mathbf{r}^{\prime}\right)=\frac{i}{4} H_{0}^{(1)}\left(\left|\mathbf{r}-\mathbf{r}^{\prime}\right|\right)
$$

Then taking advantage of the asymptotic behavior of the Hankel function, the far field for which $\mathrm{k}\left(\left|\mathrm{r}-\mathrm{r}^{\prime}\right|\right) \rightarrow \infty$ can be expressed as:

$$
E_{z}^{s}(\mathbf{r})=\frac{i}{4}\left(\frac{2}{\pi k r}\right)^{1 / 2} \exp [i(k r+3 \pi / 4)] \times \oint\left[i k(\hat{\mathbf{n}} \cdot \hat{\mathbf{r}}) E_{z}^{s}\left(\mathbf{r}^{\prime}\right)+\frac{\partial E_{z}^{s}\left(\mathbf{r}^{\prime}\right)}{\partial n}\right] \times \exp \left(-i k \hat{\mathbf{r}} \cdot \mathbf{r}^{\prime}\right) d r^{\prime}
$$

Using the boundary condition of Maxwell's equations at the surface of the dielectric particle, the spatial derivative term of the electric field is expressed in terms of linear terms involving the magnetic field:

$E_{z}^{s}(\mathbf{r})=\frac{k}{4}\left(\frac{2}{\pi k r}\right)^{1 / 2} \exp [i(k r+3 \pi / 4)] \times \oint\left[n_{x} H_{y}^{s}\left(\mathbf{r}^{\prime}\right)-n_{y} H_{x}^{s}\left(\mathbf{r}^{\prime}\right)-(\hat{\mathbf{n}} \cdot \hat{\mathbf{r}}) E_{z}^{s}\left(\mathbf{r}^{\prime}\right)\right] \times \exp \left(-i k \hat{\mathbf{r}} \cdot \mathbf{r}^{\prime}\right) d r^{\prime}$

It follows that the nonzero phase matrix elements $P_{11}, P_{12}, P_{33}$ and $P_{34}$ are:

$$
\begin{gathered}
P_{11}(\hat{s})=\left\lfloor\left. F_{\mathrm{TM}}(\hat{s})\right|^{2}+\left|F_{\mathrm{TE}}(\hat{s})\right|^{2}\right] / 2, \\
\left.P_{12}(\hat{s})=\left.|| F_{\mathrm{TM}}(\hat{s})\right|^{2}-\left|F_{\mathrm{TE}}(\hat{s})\right|^{2}\right] / 2, \\
P_{33}(\hat{s})=\operatorname{Re}\left\{F_{\mathrm{TM}}(\hat{s}) \cdot\left[F_{\mathrm{TE}}(\hat{s})\right]^{*}\right\}, \\
P_{34}(\hat{s})=\operatorname{Im}\left\{F_{\mathrm{TM}}(\hat{s}) \cdot\left[F_{\mathrm{TE}}(\hat{s})\right]^{*}\right\}, \\
F_{\mathrm{TM}}(\hat{s})=\frac{k}{4} \oint\left[n_{x} H_{y}^{s}\left(\mathbf{r}^{\prime}\right)-n_{y} H_{x}^{s}\left(\mathbf{r}^{\prime}\right)-(\hat{n} \cdot \hat{s}) E_{z}^{s}\left(\mathbf{r}^{\prime}\right)\right] \cdot \operatorname{eps}\left(-i k \hat{s} \cdot \mathbf{r}^{\prime}\right) d r^{\prime}, \\
F_{\mathrm{TE}}(\hat{s})=\frac{k}{4} \oint\left[n_{x} E_{y}^{s}\left(\mathbf{r}^{\prime}\right)-n_{y} E_{x}^{s}\left(\mathbf{r}^{\prime}\right)+(\hat{n} \cdot \hat{s}) H_{z}^{s}\left(\mathbf{r}^{\prime}\right)\right] \cdot \operatorname{eps}\left(-i k \hat{s} \cdot \mathbf{r}^{\prime}\right) d r^{\prime},
\end{gathered}
$$


where $\hat{s}$ is the unit vector in the scattering direction, and $n_{x}$ and $n_{y}$ are the $\mathrm{x}$ and $\mathrm{y}$ surface normal vector components, respectively. The asterisk here denotes the complex conjugate. The scattered fields used in calculating $F_{\mathrm{TM}}$ and $F_{\mathrm{TE}}$ are the relative complex amplitudes to the incident field amplitudes. 


\section{EXTENSION OF THE DISCONTINUOUS GALERKIN METHOD TO 3-}

\section{DIMENSIONAL CALCULATIONS OF SINGLE PARTICLE OPTICAL PROPERTIES}

The DG method gives excellent simulations of single particle optical properties in

2-dimensional geometry. We want to find out how well it works for the 3-dimensional (3D) calculation.

\subsection{Semi-discrete form of the governing equations for the near field calculation}

Using Language polynomials as shape functions and test functions, the discrete form of the governing equations in 3-D geometry is derived from vector equations (3.19). In the particle and "free space" regions,

$$
\begin{aligned}
& \frac{\partial \widetilde{H}_{x}^{s}}{\partial t}=\mathbf{M}^{-1} \mathbf{S}^{z} \widetilde{E}_{y}^{s}-\mathbf{M}^{-1} \mathbf{S}^{y} \widetilde{E}_{z}^{s}+\mathbf{M}^{-1} \mathbf{C} \widetilde{F}_{H x}^{s}, \\
& \frac{\partial \widetilde{H}_{y}^{s}}{\partial t}=\mathbf{M}^{-1} \mathbf{S}^{x} \widetilde{E}_{z}^{s}-\mathbf{M}^{-1} \mathbf{S}^{z} \widetilde{E}_{x}^{s}+\mathbf{M}^{-1} \mathbf{C} \widetilde{F}_{H y}^{s}, \\
& \frac{\partial \widetilde{H}_{z}^{s}}{\partial t}=\mathbf{M}^{-1} \mathbf{S}^{y} \widetilde{E}_{x}^{s}-\mathbf{M}^{-1} \mathbf{S}^{x} \widetilde{E}_{y}^{s}+\mathbf{M}^{-1} \mathbf{C} \widetilde{F}_{H z}^{s} \\
& \varepsilon \frac{\partial \widetilde{E}_{x}^{s}}{\partial t}=\mathbf{M}^{-1} \mathbf{S}^{y} \widetilde{H}_{z}^{s}-\mathbf{M}^{-1} \mathbf{S}^{z} \widetilde{H}_{y}^{s}+\mathbf{M}^{-1} \mathbf{C} \widetilde{F}_{E x}^{s}+(1-\varepsilon) \frac{\partial \widetilde{E}_{x}^{i}}{\partial t}-\sigma\left(\widetilde{E}_{x}^{s}+\widetilde{E}_{x}^{i}\right) . \\
& \varepsilon \frac{\partial \widetilde{E}_{y}^{s}}{\partial t}=\mathbf{M}^{-1} \mathbf{S}^{z} \widetilde{H}_{x}^{s}-\mathbf{M}^{-1} \mathbf{S}^{x} \widetilde{H}_{z}^{s}+\mathbf{M}^{-1} \mathbf{C} \widetilde{F}_{E y}^{s}+(1-\varepsilon) \frac{\partial \widetilde{E}_{y}^{i}}{\partial t}-\sigma\left(\widetilde{E}_{y}^{s}+\widetilde{E}_{y}^{i}\right) \\
& \varepsilon \frac{\partial \widetilde{E}_{z}^{s}}{\partial t}=\mathbf{M}^{-1} \mathbf{S}^{x} \widetilde{H}_{y}^{s}-\mathbf{M}^{-1} \mathbf{S}^{y} \widetilde{H}_{x}^{s}+\mathbf{M}^{-1} \mathbf{C} \widetilde{F}_{E z}^{s}+(1-\varepsilon) \frac{\partial \widetilde{E}_{z}^{i}}{\partial t}-\sigma\left(\widetilde{E}_{z}^{s}+\widetilde{E}_{z}^{i}\right)
\end{aligned}
$$

In the PML region: 


$$
\begin{aligned}
& \frac{\partial \widetilde{C}_{x}^{s}}{\partial t}=\mathbf{M}^{-1} \mathbf{S}^{z} \widetilde{E}_{y}^{s}-\mathbf{M}^{-1} \mathbf{S}^{y} \widetilde{E}_{z}^{s}+\mathbf{M}^{-1} \mathbf{C} \widetilde{F}_{H x}^{s}-\sigma_{z} \widetilde{C}_{x}^{s}, \\
& \frac{\partial \widetilde{C}_{y}^{s}}{\partial t}=\mathbf{M}^{-1} \mathbf{S}^{x} \widetilde{E}_{z}^{s}-\mathbf{M}^{-1} \mathbf{S}^{z} \widetilde{E}_{x}^{s}+\mathbf{M}^{-1} \mathbf{C} \widetilde{F}_{H y}^{s}-\sigma_{x} \widetilde{C}_{y}^{s}, \\
& \frac{\partial \widetilde{C}_{z}^{s}}{\partial t}=\mathbf{M}^{-1} \mathbf{S}^{y} \widetilde{E}_{x}^{s}-\mathbf{M}^{-1} \mathbf{S}^{x} \widetilde{E}_{y}^{s}+\mathbf{M}^{-1} \mathbf{C} \widetilde{F}_{E z}^{s}-\sigma_{y} \widetilde{C}_{z}^{s} \\
& \frac{\partial \widetilde{D}_{x}^{s}}{\partial t}=\mathbf{M}^{-1} \mathbf{S}^{y} \widetilde{H}_{z}^{s}-\mathbf{M}^{-1} \mathbf{S}^{z} \widetilde{H}_{y}^{s}+\mathbf{M}^{-1} \mathbf{C} \widetilde{F}_{E x}^{s}-\sigma_{z} \widetilde{D}_{x}^{s} \\
& \frac{\partial \widetilde{D}_{y}^{s}}{\partial t}=\mathbf{M}^{-1} \mathbf{S}^{z} \widetilde{H}_{x}^{s}-\mathbf{M}^{-1} \mathbf{S}^{x} \widetilde{H}_{z}^{s}+\mathbf{M}^{-1} \mathbf{C} \widetilde{F}_{E y}^{s}-\sigma_{x} \widetilde{D}_{y}^{s} \\
& \frac{\partial \widetilde{D}_{z}^{s}}{\partial t}=\mathbf{M}^{-1} \mathbf{S}^{x} \widetilde{H}_{y}^{s}-\mathbf{M}^{-1} \mathbf{S}^{y} \widetilde{H}_{x}^{s}+\mathbf{M}^{-1} \mathbf{C} \widetilde{F}_{E z}^{s}-\sigma_{y} \widetilde{D}_{z}^{s},
\end{aligned}
$$

$\frac{\partial \widetilde{H}_{x}^{s}}{\partial t}=\mathbf{M}^{-1} \mathbf{S}^{z} \widetilde{E}_{y}^{s}-\mathbf{M}^{-1} \mathbf{S}^{y} \widetilde{E}_{z}^{s}+\mathbf{M}^{-1} \mathbf{C} \widetilde{F}_{H x}^{s}+\left(\sigma_{x}-\sigma_{z}\right) \widetilde{C}_{x}^{s}-\sigma_{y} \widetilde{H}_{x}^{s}$,

$$
\frac{\partial \widetilde{H}_{y}^{s}}{\partial t}=\mathbf{M}^{-1} \mathbf{S}^{x} \widetilde{E}_{z}^{s}-\mathbf{M}^{-1} \mathbf{S}^{z} \widetilde{E}_{x}^{s}+\mathbf{M}^{-1} \mathbf{C} \widetilde{F}_{H y}^{s}+\left(\sigma_{y}-\sigma_{x}\right) \widetilde{C}_{y}^{s}-\sigma_{z} \widetilde{H}_{y}^{s},
$$$$
\frac{\partial \widetilde{H}_{z}^{s}}{\partial t}=\mathbf{M}^{-1} \mathbf{S}^{y} \widetilde{E}_{x}^{s}-\mathbf{M}^{-1} \mathbf{S}^{x} \widetilde{E}_{y}^{s}+\mathbf{M}^{-1} \mathbf{C} \widetilde{F}_{H z}^{s}+\left(\sigma_{z}-\sigma_{y}\right) \widetilde{C}_{z}^{s}-\sigma_{x} \widetilde{H}_{z}^{s}
$$$$
\frac{\partial \widetilde{E}_{x}^{s}}{\partial t}=\mathbf{M}^{-1} \mathbf{S}^{y} \widetilde{H}_{z}^{s}-\mathbf{M}^{-1} \mathbf{S}^{z} \widetilde{H}_{y}^{s}+\mathbf{M}^{-1} \mathbf{C} \widetilde{F}_{E x}^{s}+\left(\sigma_{x}-\sigma_{z}\right) \widetilde{D}_{x}^{s}-\sigma_{y} \widetilde{E}_{x}^{s}
$$$$
\frac{\partial \widetilde{E}_{y}^{s}}{\partial t}=\mathbf{M}^{-1} \mathbf{S}^{z} \widetilde{H}_{x}^{s}-\mathbf{M}^{-1} \mathbf{S}^{x} \widetilde{H}_{z}^{s}+\mathbf{M}^{-1} \mathbf{C}_{E y}^{s}+\left(\sigma_{y}-\sigma_{x}\right) \widetilde{D}_{y}^{s}-\sigma_{z} \widetilde{E}_{y}^{s}
$$$$
\frac{\partial \widetilde{E}_{z}^{s}}{\partial t}=\mathbf{M}^{-1} \mathbf{S}^{x} \widetilde{H}_{y}^{s}-\mathbf{M}^{-1} \mathbf{S}^{y} \widetilde{H}_{x}^{s}+\mathbf{M}^{-1} \mathbf{C} \widetilde{F}_{E z}^{s}+\left(\sigma_{z}-\sigma_{y}\right) \widetilde{D}_{z}^{s}-\sigma_{x} \widetilde{E}_{z}^{s},
$$ 
where $\mathbf{M}, \quad \mathbf{S}^{\mathrm{x}}, \quad \mathbf{S}^{\mathrm{y}}, \quad \mathbf{S}^{\mathrm{z}}$ are $N_{p} \times N_{p}$ matrices and $\mathbf{C}$ is a $N_{p} \times 4 N_{f p}$ matrix. $N_{p}=(N+1)(N+2)(N+3) / 6$ is the number of nodes and $N_{\mathrm{fp}}=(N+1)(N+2) / 2$ is the number of nodes at each face of an triangular element. These matrices are defined as:

$$
\begin{aligned}
& \mathbf{M}_{i j}=\iiint_{D_{k}} l_{j}(x, y, z) l_{i}(x, y, z) d x d y d z, \\
& \mathbf{S}_{i j}^{x}=\iiint_{D_{k}} \frac{\partial l_{j}(x, y, z)}{\partial x} l_{i}(x, y, z) d x d y d z, \\
& \mathbf{S}_{i j}^{y}=\iiint_{D_{k}} \frac{\partial l_{j}(x, y, z)}{\partial y} l_{i}(x, y, z) d x d y d z, \\
& \mathbf{S}_{i j}^{z}=\iiint_{D_{k}} \frac{\partial l_{j}(x, y, z)}{\partial z} l_{i}(x, y, z) d x d y d z \\
& \mathbf{C}_{i s}=\oint_{\partial D_{k}} l_{j_{s}}(x, y, z) l_{i}(x, y, z) d s .
\end{aligned}
$$

where $l_{i}(x, y, z)$ is the language polynomial associated with the $i^{\text {th }}$ node of the element, $D_{k}$ denotes the $k^{\text {th }}$ triangular element, and $j_{s}$ denotes the index of a node on the surface (with the nodes on the vertices counted three times and the other nodes on the edges counted twice). The numerical fluxes are derived from the vector form Eq. (3.17), using upwind flux which means that $\alpha=1$ :

$$
\begin{aligned}
\widetilde{F}_{H x}^{s}= & \frac{1}{Y^{-}+Y^{+}}\left\{\left[\hat{n}_{y}\left(E_{z}^{s-}-E_{z}^{s+}\right)-\hat{n}_{z}\left(E_{y}^{s-}-E_{y}^{s+}\right)\right] Y^{+}\right. \\
& \left.+\hat{n}_{x}\left[\hat{n}_{x}\left(H_{x}^{s-}-H_{x}^{s+}\right)+\hat{n}_{y}\left(H_{y}^{s-}-H_{y}^{s+}\right)+\hat{n}_{z}\left(H_{z}^{s-}-H_{z}^{s+}\right)\right]-\left(H_{x}^{s-}-H_{x}^{s+}\right)\right\} \\
\widetilde{F}_{H y}^{s}= & \frac{1}{Y^{-}+Y^{+}}\left\{\left[\hat{n}_{z}\left(E_{x}^{s-}-E_{x}^{s+}\right)-\hat{n}_{x}\left(E_{z}^{s-}-E_{z}^{s+}\right)\right] Y^{+}\right. \\
& \left.+\hat{n}_{y}\left[\hat{n}_{x}\left(H_{x}^{s-}-H_{x}^{s+}\right)+\hat{n}_{y}\left(H_{y}^{s-}-H_{y}^{s+}\right)+\hat{n}_{z}\left(H_{z}^{s-}-H_{z}^{s+}\right)\right]-\left(H_{y}^{s-}-H_{y}^{s+}\right)\right\} \\
& \widetilde{F}_{H z}^{s}=\frac{1}{Y^{-}+Y^{+}}\left\{\left[\hat{n}_{x}\left(E_{y}^{s-}-E_{y}^{s+}\right)-\hat{n}_{y}\left(E_{x}^{s-}-E_{x}^{s+}\right)\right] Y^{+}\right. \\
& \left.+\hat{n}_{z}\left[\hat{n}_{x}\left(H_{x}^{s-}-H_{x}^{s+}\right)+\hat{n}_{y}\left(H_{y}^{s-}-H_{y}^{s+}\right)+\hat{n}_{z}\left(H_{z}^{s-}-H_{z}^{s+}\right)\right]-\left(H_{z}^{s-}-H_{z}^{s+}\right)\right\}
\end{aligned}
$$




$$
\begin{aligned}
\widetilde{F}_{E x}^{s}= & \frac{1}{Z^{-}+Z^{+}}\left\{\left[-\hat{n}_{y}\left(H_{z}^{s-}-H_{z}^{s+}\right)+\hat{n}_{z}\left(H_{y}^{s-}-H_{y}^{s+}\right)\right] Z^{+}\right. \\
& \left.+\hat{n}_{x}\left[\hat{n}_{x}\left(E_{x}^{s-}-E_{x}^{s+}\right)+\hat{n}_{y}\left(E_{y}^{s-}-E_{y}^{s+}\right)+\hat{n}_{z}\left(E_{z}^{s-}-E_{z}^{s+}\right)\right]-\left(E_{x}^{s-}-E_{x}^{s+}\right)\right\} \\
\widetilde{F}_{E y}^{s}= & \frac{1}{Z^{-}+Z^{+}}\left\{\left[-\hat{n}_{z}\left(H_{x}^{s-}-H_{x}^{s+}\right)+\hat{n}_{x}\left(H_{z}^{s-}-H_{z}^{s+}\right)\right] Z^{+}\right. \\
& \left.+\hat{n}_{y}\left[\hat{n}_{x}\left(E_{x}^{s-}-E_{x}^{s+}\right)+\hat{n}_{y}\left(E_{y}^{s-}-E_{y}^{s+}\right)+\hat{n}_{z}\left(E_{z}^{s-}-E_{z}^{s+}\right)\right]-\left(E_{y}^{s-}-E_{y}^{s+}\right)\right\} \\
\widetilde{F}_{E z}^{s}= & \frac{1}{Z^{-}+Z^{+}}\left\{\left[-\hat{n}_{x}\left(H_{y}^{s-}-H_{y}^{s+}\right)+\hat{n}_{y}\left(H_{x}^{s-}-H_{x}^{s+}\right)\right] Z^{+}\right. \\
& \left.+\hat{n}_{z}\left[\hat{n}_{x}\left(E_{x}^{s-}-E_{x}^{s+}\right)+\hat{n}_{y}\left(E_{y}^{s-}-E_{y}^{s+}\right)+\hat{n}_{z}\left(E_{z}^{s-}-E_{z}^{s+}\right)\right]-\left(E_{z}^{s-}-E_{z}^{s+}\right)\right\}
\end{aligned}
$$

where the electric and magnetic fields are associated with the fields on the nodes where the fluxes are calculated. In these equations, $\widetilde{H}_{x}^{s}, \widetilde{H}_{y}^{s}, \widetilde{H}_{z}^{s}, \widetilde{E}_{x}^{s}, \widetilde{E}_{y}^{s}, \widetilde{E}_{z}^{s}, \widetilde{C}_{x}^{s}, \widetilde{C}_{y}^{s}, \widetilde{C}_{z}^{s}$, $\widetilde{D}_{x}^{s}, \widetilde{D}_{y}^{s}$ and $\widetilde{D}_{z}^{s}$ are $N_{p} \times 1$ vectors whose values are the fields values on the nodal points which are denoted by the index of these vectors, and $\widetilde{F}_{H x}^{s}, \widetilde{F}_{H y}^{s}, \widetilde{F}_{H z}^{s}, \widetilde{F}_{E x}^{s}, \widetilde{F}_{E y}^{s}$ and $\widetilde{F}_{E z}^{s}$ are $4 N_{f p} \times 1$ vectors whose values are corresponding numerical flux on the surface nodal points which are denoted by the index of these vectors.

\subsection{Transformation of the near field to the far field}

Having the semi-discrete form equations, using the Range-Kutta scheme to integrate over a period time during which the fields go into the steady state with sinusoidal incident fields, the near fields can be calculated and transformed into frequency domain as in the 2D calculations. Using the near fields in frequency domain, the far field and the scattering phase matrix can be calculated. The transformation is derived using the Green's function method [9] and shown below: 


$$
\left(\begin{array}{ll}
S_{2} & S_{3} \\
S_{4} & S_{1}
\end{array}\right)=\left(\begin{array}{cc}
F_{/ /, x} & F_{/ /, y} \\
F_{\perp, x} & F_{\perp, y}
\end{array}\right)\left(\begin{array}{cc}
\hat{e}_{\perp} \cdot \hat{e}_{x} & \hat{e}_{\perp} \cdot \hat{e}_{y} \\
\hat{e}_{\perp} \cdot \hat{e}_{y} & -\hat{e}_{\perp} \cdot \hat{e}_{x}
\end{array}\right)
$$

where $\hat{e}_{\perp}$ is the unit vector perpendicular to the scattering plane, $\hat{e}_{x}$ and $\hat{e}_{y}$ are unit vectors that are perpendicular to the incident direction and perpendicular to each other. Applying the volume integral scheme, the matrix $F$ are expressed as:

$$
\begin{aligned}
& \left(\begin{array}{c}
F_{/ /, x} \\
F_{\perp, x}
\end{array}\right)=-\frac{i k^{3}}{4 \pi} \iiint\left[\varepsilon\left(\mathbf{r}^{\prime}\right)-1\right]\left(\begin{array}{c}
\hat{\mathbf{e}}_{/ /} \cdot \mathbf{E}\left(\mathbf{r}^{\prime}\right) \\
\hat{\mathbf{e}}_{\perp} \cdot \mathbf{E}\left(\mathbf{r}^{\prime}\right)
\end{array}\right) \exp \left(-i k \hat{\mathbf{e}} \cdot \mathbf{r}^{\prime}\right) d r^{\prime 3} \mid \\
& E_{x}^{i}=1, E_{y}^{i}=0 \\
& \left(\begin{array}{l}
F_{/ /, y} \\
F_{\perp, y}
\end{array}\right)=-\left.\frac{i k^{3}}{4 \pi} \iiint\left[\varepsilon\left(\mathbf{r}^{\prime}\right)-1\right]\left(\begin{array}{l}
\hat{\mathbf{e}}_{/ /} \cdot \mathbf{E}\left(\mathbf{r}^{\prime}\right) \\
\hat{\mathbf{e}}_{\perp} \cdot \mathbf{E}\left(\mathbf{r}^{\prime}\right)
\end{array}\right) \exp \left(-i k \hat{\mathbf{e}} \cdot \mathbf{r}^{\prime}\right) d r^{\prime 3}\right|_{E_{x}^{i}=0, E_{y}^{i}=1}
\end{aligned}
$$

where the integral is performed over the particle. Using the amplitude scattering matrix and Eq. (2.4), the scattering phase matrix can be calculated. 


\section{NUMERICAL RESULTS AND DISCUSSIONS}

The key parameters for the six DGTD calculations discussed in this section are listed in Table 1.

\subsection{Investigation of the applicability of the Discontinuous Galerkin Time- Domain method to the computation of light scattering properties of circular particles}

First, as demonstration of accuracy, the DGTD method is used to compute the scattering phase matrix of infinitely long circular cylinders with normal illumination. In Fig. 3, the left panels show the nonzero scattering phase matrix elements for a size parameter of 50 and an incident wavelength of $0.532 \mu \mathrm{m}$. The refractive index of ice crystals at this wavelength is $1.3117+\mathrm{i} 1.489 \times 10^{-9}$, which corresponds to an essentially non-absorptive case. The dotted and solid lines correspond to the DGTD and the analytical solution, respectively. The right panels show the relative differences between the DGTD solution and the analytical solution; the relative difference may get large when the true values are small. The results from the DGTD calculation agree very well with the analytical solution. For the phase function, except at a few angles, the differences between the DGTD solution and the exact solution are smaller than $10 \%$. Larger differences are observed at angles around $90^{\circ}$ and in the backscattering direction, where the true values of the phase function are relatively small. (For polarization elements, 
points where the differences are larger than $100 \%$ are not shown.) The data points of relative difference in the figure are generally smaller than $20 \%$.

The panels in Fig. 4 are as Fig. 3 except that the size parameter is now 100. The accuracy is similar to the case shown in Fig. 3. The computations were done on an IBM p5-575+ cluster, with cores running at $1.9 \mathrm{GHz}$. and Table 1 lists the CPU time necessary to complete the simulations of the circular cylinders with size parameters 50 and 100 . Since computational time is dependent on the hardware used, and relative times are the more significant data, we have listed the cpu time in multiples of the time required for the particle size 50 case. The larger particle simulation used approximately 5.5 times as much computational time as that needed for the smaller particle simulation.

To demonstrate accuracy in the absorptive case, an incident wavelength of $12 \mu \mathrm{m}$ was considered. In this case we took advantage of the fact that with higher absorbtion, the solution converges rapidly to the steady state, making finer mesh calculations possible. We reduced by half the mesh size, so that no cell had diameter larger than a quarter wavelength. Fig. 5 shows the nonzero phase matrix elements (left panels) of the infinitely long circular cylinders with a size parameter of 50 and the relative differences (right panels) between the DGTD solution and the exact solution. The refractive index is $1.2799+0.4133 \mathrm{i}$. The results for the absorptive case show a better agreement with the analytical solution, as might be expected because of the smaller mesh size, but very subtle differences remain in the backscattering direction on the polarized elements $\mathrm{P}_{12}$ and $\mathrm{P}_{34}$ at values close to zero. The largest difference between the phase functions is less than $0.2 \%$. 
Fig. 6 is the same as Fig. 5 except that the size parameter is 100. The pattern of the phase matrix and the accuracy show similar features of the previous case with a size parameter of 50. It is evident from a comparison between Figs. 5 and 6 with Figs. 3 and 4 that the accuracy for the phase matrices is considerably increased when the mesh size is decreased.

\subsection{Application of the Discontinuous Galerkin Time-Domain method to the computation of light scattering properties of hexagonal ice crystal}

In the case of hexagonal geometry, the scattered field will depend on the orientation of the particle with respect to the direction of propagation of the incident wave. In applications, where there will be many scatterers, information on single orientations is not as important as the average over all orientations. Because of the 60 degree rotational symmetry of the crystal, we need only consider a range of orientations in an angular interval of 60 degrees. For the calculations in this section, 30 orientations were calculated, at 2 degree intervals. Cpu times in Table 1 are accordingly recorded as multiples of 30 .

The nonzero phase matrix elements of 2-D hexagonal ice crystals with size parameters of 50 and 100, and an incident wavelength of $0.532 \mu \mathrm{m}$ are calculated and shown in Figs. $\mathbf{7}$ and $\mathbf{8}$, respectively. The results are compared with those calculated from the IGOM [30] algorithm. The phase function shows a $22^{\circ}$ halo peak and a scattering maximum at round $150^{\circ}$. 
Fig. 7 shows the nonzero phase matrix elements computed from the DGTD and IGOM for randomly oriented hexagonal geometry with a size parameter of 50 . The $22^{\circ}$ halo peak in the P11 element (i.e., the phase function), which stems (as shown below) from the rays that undergo two sequential refractions, is quite pronounced. A sharp peak at $\sim 10^{\circ}$ is due to diffraction at this size parameter. Overall, the DGTD and IGOM results for the phase function are consistent, although noticeable differences occur at large scattering angles, e.g., near $156^{\circ}$. For the other phase matrix elements, large errors appear in the IGOM results in comparison with the DGTD counterparts, particularly, in the case of $\mathrm{P} 34 / \mathrm{P} 11$. Recalll that $\mathrm{P} 12 / \mathrm{P} 11$. $\mathrm{P} 33 / \mathrm{P} 11$ and $\mathrm{P} 34 / \mathrm{P} 11$ are associated with the polarization state of the scattered waves and are sensitive to the interference of the scattered waves. As an approximation, IGOM cannot well simulate this interference effect at large scattering angles.

Fig. 8 is similar to Fig. 7, except for a size parameter of 100. Evidently, with an increase in the size parameter, the agreement between the IGOM and DGTD increases in the case of the phase function. Additionally, a pronounced scattering maximum at $156^{\circ}$ is noticeable, which is attributed mainly to the $5^{\text {th }}$ order rays, as illustrated by Cai and Liou [31]. Substantial errors of IGOM are still noticed at a size parameter of 100 for the other phase matrix elements, particularly in the case of P34/P11. (Note that Cai and Liou used the conventional geometric optics version of the ray-tracing technique in their simulation, and their numbering order is different.) Many detailed features in the phase function and polarization-related phase matrix elements noticed in the present results are not observed in the counterparts reported in [31]. 
Fig. 9 shows the contributions of the rays of various orders to the normalized phase function of the 2-D randomly oriented hexagonal ice crystals with a size parameter of 100 and an incident wavelength of $0.532 \mu \mathrm{m}$. The $0+1$ (reflection + diffraction) curve indicates the contribution of the diffraction and the external reflection. (In the following discussion, the reader may want to look ahead to the side panels of Fig. $\mathbf{1 0}$ for clarification of the numbering.) The $0+1+2$ curve indicates the sum of the $0+1$ contributions and the contribution of the rays that undergo two refractions without internal reflection, the $0+1+2+3$ curve indicates the sum of the $0+1+2$ contributions and the contribution of rays that undergo two refractions and one internal reflection. It is evident that the $22^{\circ}$ halo is mainly due to the rays that undergo two refractions without internal reflection. The phase function at angles between $40^{\circ}$ and $140^{\circ}$ is due mainly to contributions from the third order rays that undergo two refractions and one internal reflection. The weak peak between $140^{\circ}$ and $160^{\circ}$ is almost entirely due to rays of order 4 (two internal reflections) and order 5 (three internal reflections). The backscattering was contributed by a combination of higher order rays. For forward scattering, diffraction contributes the largest fraction, whereas the $2^{\text {nd }}$ and higher order rays contribute only a small amount.

The results in Figs. 5-9 are all based on averages over crystal orientation. An intriguing structure is revealed by displaying the scattering data without averaging. The upper panel of Fig. 10 details the scattering phase function with respect to the incident and scattering angles, which are computed from the geometric optics method. Note that these results are for specific orientations of ice crystals. The incident and scattering configuration is shown in the lower panel of Fig. 10. As noted above, the rotational 
symmetry of the ice crystals means that a $60^{\circ}$ range of incident angles is sufficient, but it is visually useful to show data over a $120^{\circ}$ range. No extra computations were done: the results between $60^{\circ}$ and $120^{\circ}$ are simply a duplication of those between $0^{\circ}$ and $60^{\circ}$. The two off-center wavy bands of strong scattering show that the $22^{\circ}$ halo is observed in with respect to almost all orientation angles, a feature which can also be seen clearly in the averaged results presented in Fig. 8 .

In addition to the $22^{\circ}$ halo peak, other interesting features are evident. The striking diagonally tilted lines of high amplitude for the phase function are comprised of contributions from nearly all scattering angles and stem mainly from the external reflection. These maximum lines do not leave a trace in the curve of phase function shown in Fig. 9, which is averaged over incident angles, because the phase function amplitude contributed by these maximum lines distributes almost uniformly with respect to the scattering angle in the average over the incident angle. It is also clear that the maximum that appears about $150^{\circ}$ in Fig. 8 is due to a strong contribution from a band incident angles around $20^{\circ}$, with secondary bands between $40^{\circ}$ and $60^{\circ}$.

The results from the IGOM calculations may also be displayed in the manner of Fig. 10, and they indicate many of the same structural features as seen in the DG results. In fact, some indication of the origin of these features may be seen by constructing a succession of such two dimensional displays, with successively adding higher and higher orders of approximation (higher numbers of internal reflection). This is shown in Fig. 11, which illustrates how the major structural features seen in Fig. 10 are built up, starting with reflection, reflection plus diffraction, and subsequently adding higher numbers of 
internal reflection $(0,1,2, \ldots 9)$. The lower right-hand panel of Fig. 11 is what should be compared with the DG calculation in Fig. 10.

While the IGOM method has the advantage that such decompositions are possible, the comparison just mentioned gives us the impression that the DG representation may be the more accurate one. This impression is hard to justify without knowledge of an exact solution, or a calculation by an independent method. But with regard to some of the fine structure, the IGOM results have the appearance of being a smeared version of the DG results, and some features are missing altogether in the IGOM results. This difference is not easy to explain in terms of inadequate sampling. Each of the calculations used the same number of orientations. The IGOM calculations were done using 3000 rays per orientation, and doubling the number of rays per orientation did not noticeably change the results, nor did increasing the order of the approximation beyond 9 internal reflections.

\subsection{Applicability of the Discontinuous Galerkin Time-Domain method to scattering problems in 3-dimensional geometry}

Fig. 12 shows a tetrahedron grid used in the calculation of the optical properties of a spherical particle. The green, red and blue regions denote the particle, free space and the UPML regions, respectively. As a test we show the $\mathrm{E}_{\mathrm{x}}$ value at a chosen location in the free space with respect to the non-dimensional time in Fig. 13. The size parameter of the sphere is 3 and the refractive index is 1.313 . It is seen that the field goes into steady state after the non-dimensional time 10 . The scattering phase matrix will be able to be given using the near field and a volume integral scheme. 


\section{SUMMARY}

The DGTD method was applied to the scattering of light by dielectric particles in two-dimensional geometry. Comparison between the DGTD and the exact solutions for the scattering matrix of infinitely long circular cylinders solutions shows that the DGTD method is quite accurate. As an example of a case of non-symmetric particles, the singlescattering properties of 2-D randomly oriented hexagonal ice crystals were calculated using the DGTD method with relatively large size parameters. The comparison between results calculated from the DGTD and the geometric optics method also showed good agreement, and some of the differences were attributed to known weaknesses of the geometric optics approximation. A demonstration was also given of an intricate structure in the scattering amplitudes when considered as functions of both particle orientation and scattering angle. While we cannot currently explain many features of the structure revealed, we have the impression that geometrical optics method may not be able to capture it as well as the DG method. 


\section{REFERENCES}

1. T. Wriedt, "A review of elastic light scattering theories," Part. Part. Syst. Charact. 15, 67-74 (1998).

2. A. Kokhanovsky, Optics of Light Scattering Media (Wiley, 1999).

3. M. I. Mishchenko, W. J. Wiscombe, J. W. Hovenier and L. D. Travis, "Overview of scattering by nonspherical particles" in Light Scattering by Nonspherical Particles: Theory, Measurements, and Geophysical Applications, M. I. Mishchenko, J. W. Hovenier and L. D. Travis, eds. (Academic, 2000), pp. 29-60.

4. F. M. Kahnert, "Numerical methods in electromagnetic scattering theory," J. Quant. Spectr. Rad. Trans. 79-80, 775-824 (2003).

5. P. C. Waterman, "Symmetry, unitarity, and geometry in electromagnetic scattering," Phys. Rev. D 3, 825-839, (1971).

6. M. I. Mishchenko, L. D. Travis and D. W. Mackowski, "T-matrix computations of light scattering by nonspherical particles: a review,” J. Quant. Spectr. Rad. Trans. 55, $535-575$ (1996).

7. S. K. Yee, "Numerical solution of initial boundary value problems involving Maxwell's equations in isotropic media," IEE Trans. Antennas Propag. 14, 302-307 (1966).

8. A. Taflove and S. C. Hagness, Computational Electromagnetics 2nd ed. (Artech, 2000).

9. P. Yang, K. N. Liou, "Finite-difference time domain method for light scattering by small ice crystals in three-dimensional space," J. Opt. Soc. Am. A 13, 2072-2085 (1996). 
10. W. Sun, Q. Fu and Z. Chen, "Finite-difference time-domain solution of light scattering by dielectric particles with perfectly matched layer absorbing boundary conditions," Appl. Opt. 38, 3141-3151 (1999).

11. Q. H. Liu, "The PSTD algorithm: A time-domain method requiring only two cells per wavelength," Microw. Opt. Technol. Lett. 15, 158-165 (1997).

12. Q. H. Liu, "The pseudospectral time-domain (PSTD) algorithm for acoustic waves in absorptive media," IEEE Trans. Ultraso. Ferroelect. Freq. Contr. 45, 1044-1055 (1998).

13. G. Chen, "Modeling of the optical properties of nonspherical particles in the atmosphere," Ph. D dissertation (Department of Atmospheric Sciences, Texas A\&M University, College Station, TX, 2007).

14. E. M. Purcell and C. R. Pennypacker, "Scattering and absorption of light by nonspherical dielectric grains,” Astrophys. J. 186, 705-714 (1973).

15. B. T. Draine and P. J. Flatau, "Discrete dipole approximation for scattering calculations,” J. Opt. Soc. Am. A 11, 1491-1499 (1994).

16. M. A. Yurkin and A. G. Hoekstra, "The discrete dipole approximation: an overview and recent developments," J. Quant. Spectrosc. Radiat. Trans. 106, 558-589 (2007).

17. I. Perugia and D. Schötzau, "The hp-local discontinuous Galerkin method for lowfrequency time-harmonic Maxwell Equations,” Math. Comput., 72, 1179-1214 (2002).

18. P. Houston, I. Perugia and D. Schötzau, "Mixed discontinuous Galerkin approximation of the Maxwell Operator," SIAM J. Numer. Anal. 42, 434-450 (2004).

19. J. Hesthaven and T. Warburton, "Nodal high-order methods on unstructured Grids. I. Time-domain solution of Maxwell's equations," J. Comput. Phys. 181, 186-221 (2002). 
20. B. Cockburn, G. Karniadakis, and C.-W. Shu, "The development of discontinuous Galerkin methods," in Discontinuous Galerkin Methods: Theory, Computation and Applications, B. Cockburn, G. Karniadakis, C.-W. Shu, eds., Lecture Notes in Computational Science and Engineering (Springer, 2000), Vol. 11, pp. 3-50.

21. J. S. Hesthaven and T. Warburton, Nodal Discontinuous Galerkin Methods, Algorithms, Analysis, and Applications (Springer, 2008).

22. A. C. Cangellaris and D. B. Wright, "Analysis of the numerical error caused by the stair-stepped approximation of a conducting boundary in FDTD simulations of electromagnetic phenomena," IEEE Trans. Antennas Propagat. 39, 1518-1525 (1991).

23. M. H. Carpenter, C. A. Kennedy, Fourth-order-2N-storage Runge-Kutta schemes, TM 109112, NASA, Langley Research Center, VA, 1994.

24. S. D. Gedney, "An Anisotropic Perfectly Matched Layer-Absorbing Medium for the Truncation of FDTD lattices," IEEE Trans. Antennas Propagat. 44, 1630-1639 (1996).

25. C. F. Bohren and D. R. Huffman, Absorption and Scattering of Light by Small Particles (Wiley, 1983).

26. H.C. van de Hulst, Light Scattering by Small Particles (Dover, 1981).

27. P.-O. Persson and G. Strang, “A Simple Mesh Generator in Matlab,” SIAM Rev. 46, 329-345 (2004).

28. W. J. Gordon and C. A. Hall, "Construction of curvilinear co-ordinate systems and application to mesh generation,” Int. J. Numer. Meth. Engng. 7, 461-477 (1973).

29. E. F. Toro, Riemann Solvers and Numerical Methods for Fluid Dynamics: A Practical Introduction 2nd ed. (Springer, 1999). 
30. P. Yang and K.N. Liou, "Light scattering by hexagonal ice crystals: comparison of finite-difference time domain and geometric optics models," J. Opt. Soc. Am. A 12, $162-176(1995)$.

31. Q. Cai, K. N. Liou, "Polarized light scattering by hexagonal ice crystals: theory," Appl. Opt. 21, 3569-3580 (1982). 


\section{APPENDIX}

Table 1. Set of DGTD simulations and the computer time consumed. All calculations done on an IBM p5-575+ cluster, and only the first time reported is in hours. The remaining times are recorded as multiples of this time.

\begin{tabular}{|c|c|c|c|}
\hline Simulations & $\mathrm{Ka}, \lambda(\mu \mathrm{m})$ & \# elements, mesh size & CPU time \\
\hline \multirow{4}{*}{ DGTD for circle } & $50, \quad 0.532$ & $11119, \quad \lambda / 2$ & 1 ( $\sim 2.75$ hours $)$ \\
\hline & $100, \quad 0.532$ & $27767, \quad \lambda / 2$ & 5.45 \\
\hline & $\begin{array}{ll}50, & 12.0\end{array}$ & $27767, \lambda / 4$ & 5.45 \\
\hline & $100,12.0$ & $45458, \quad \lambda / 4$ & 12.9 \\
\hline \multirow{2}{*}{ DGTD for hexagon } & $50, \quad 0.532$ & $11147, \quad \lambda / 2$ & $1 \times 30$ \\
\hline & $100,0.532$ & $27825, \quad \lambda / 2$ & $5.45 \times 30$ \\
\hline
\end{tabular}




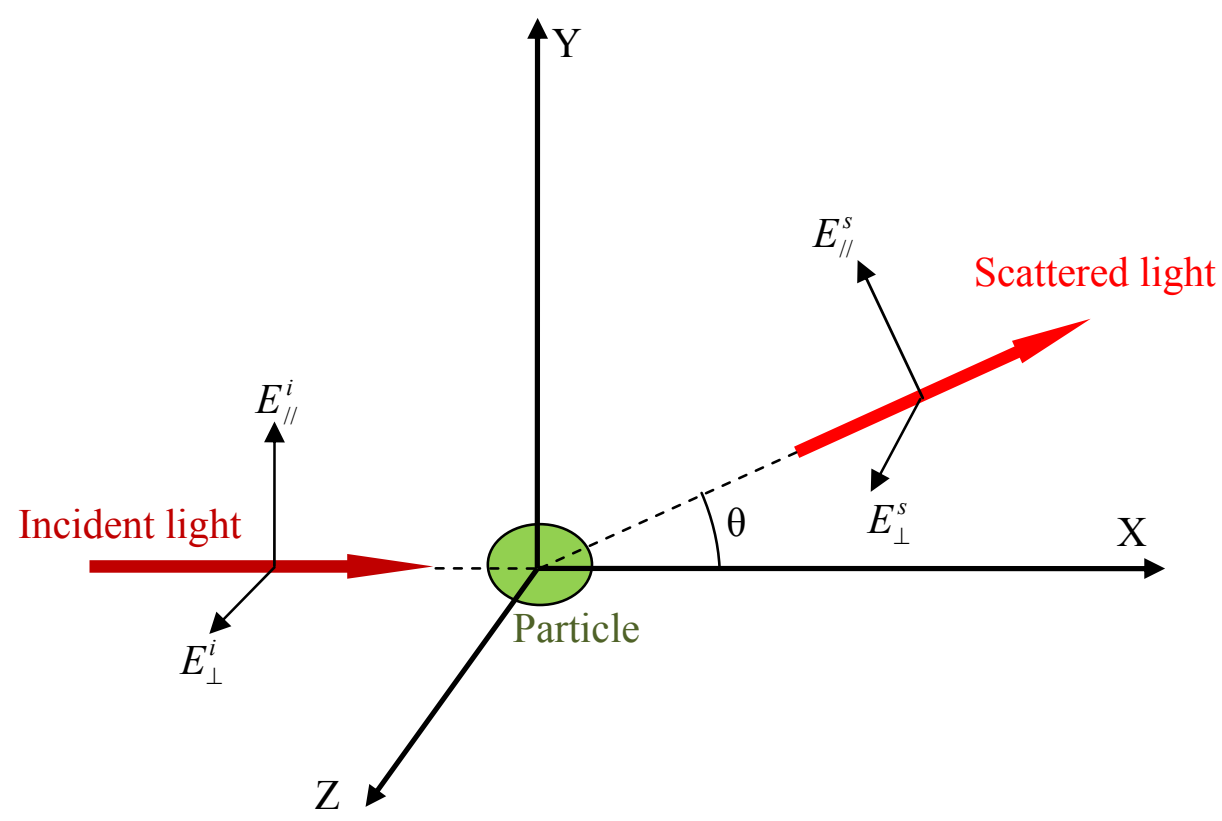

Fig. 1. Geometry of light scattering process of a particle 
(a)

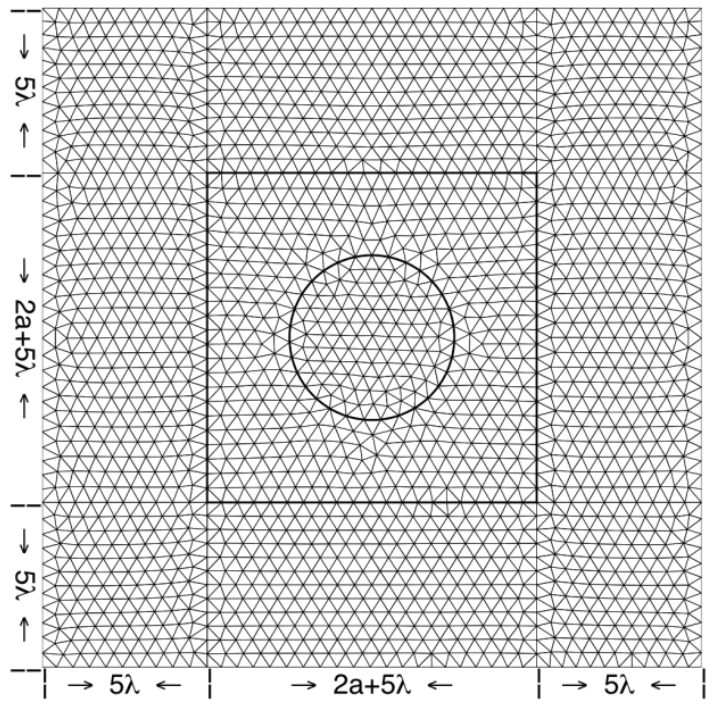

(b)

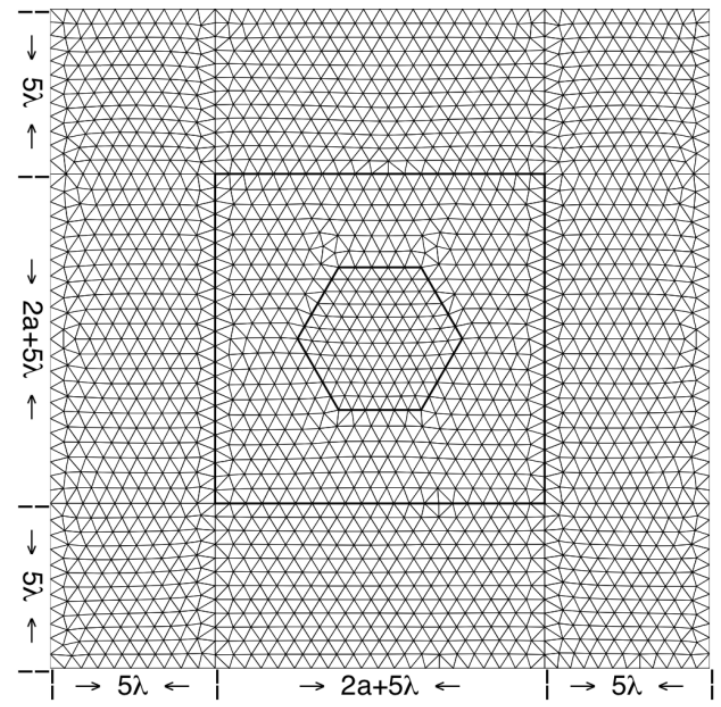

Fig. 2. Left: Grid for the simulation of an infinitely long circular cylinder. Right: Grid for the simulation of a hexagon. The size parameters are both 10. (Note: the sizes are expressed in dimensional units.) 

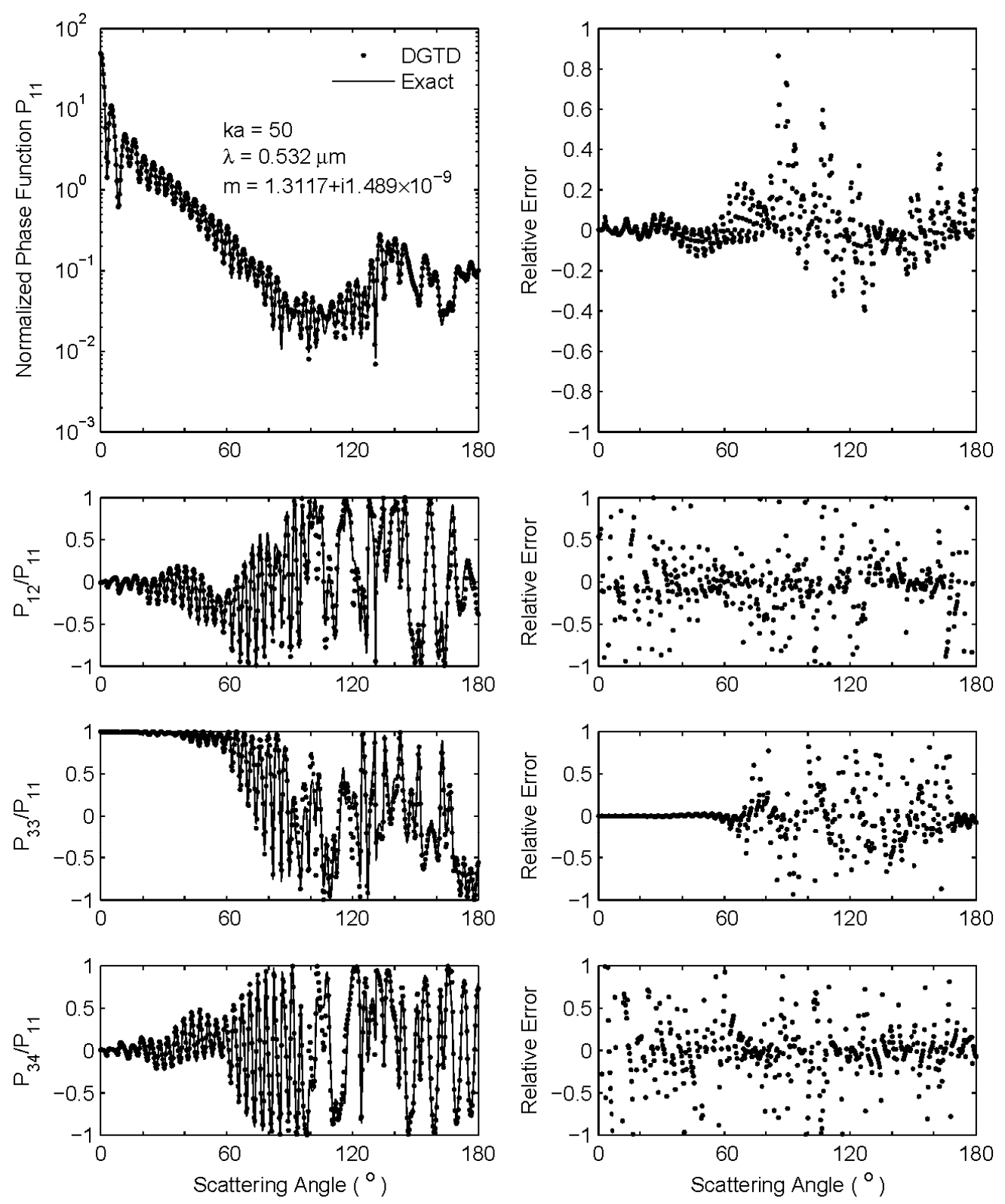

Fig. 3. Left Panel: Nonzero phase matrix elements of an infinitely long circular cylinder with a size parameter of 50 and an incident wavelength of $0.532 \mu \mathrm{m}$. 

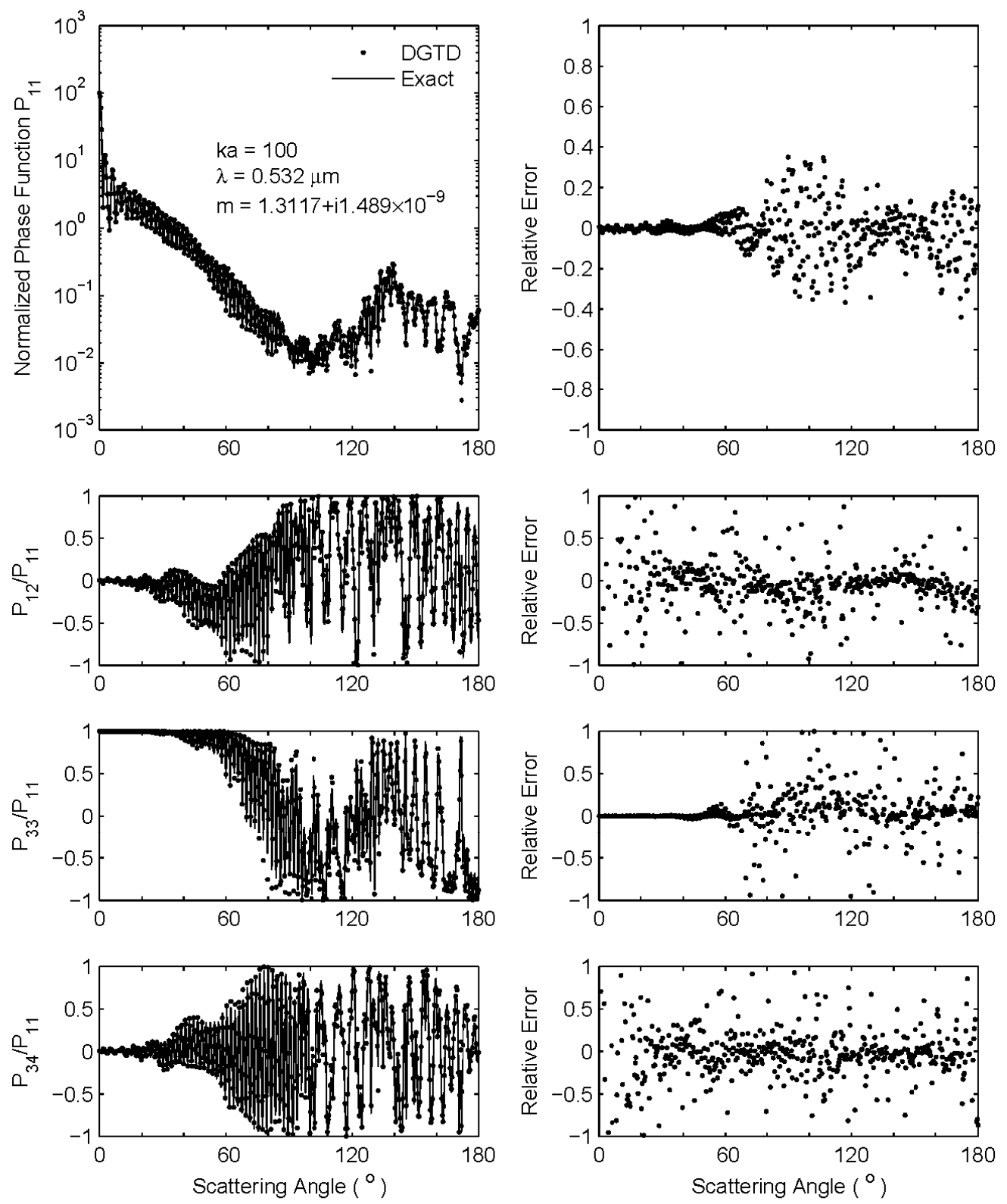

Fig. 4. Same as Fig. 3 except that the size parameter is 100. 

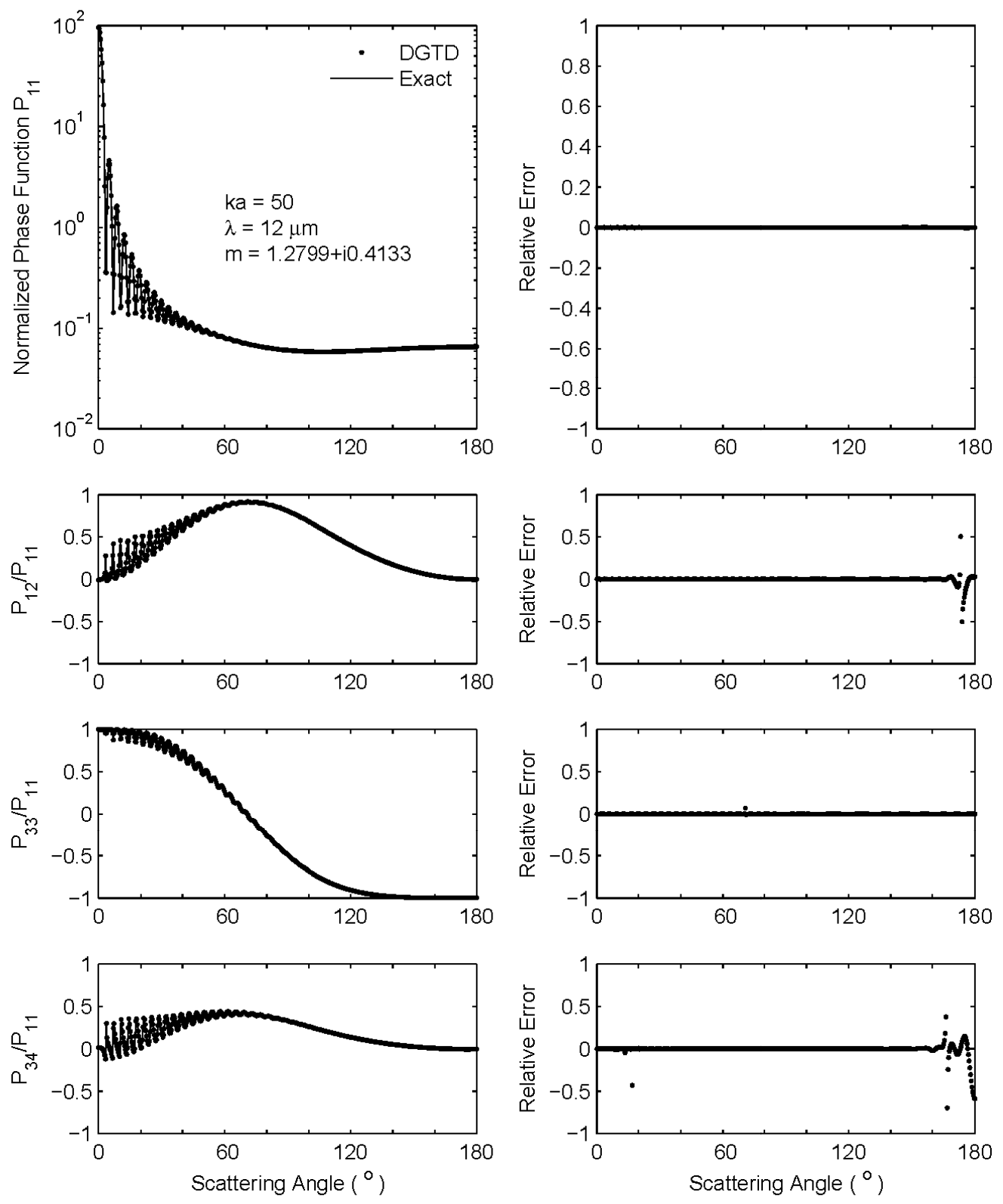

Fig. 5. The same as Fig. 3 except that the incident wavelength is $12 \mu \mathrm{m}$. 

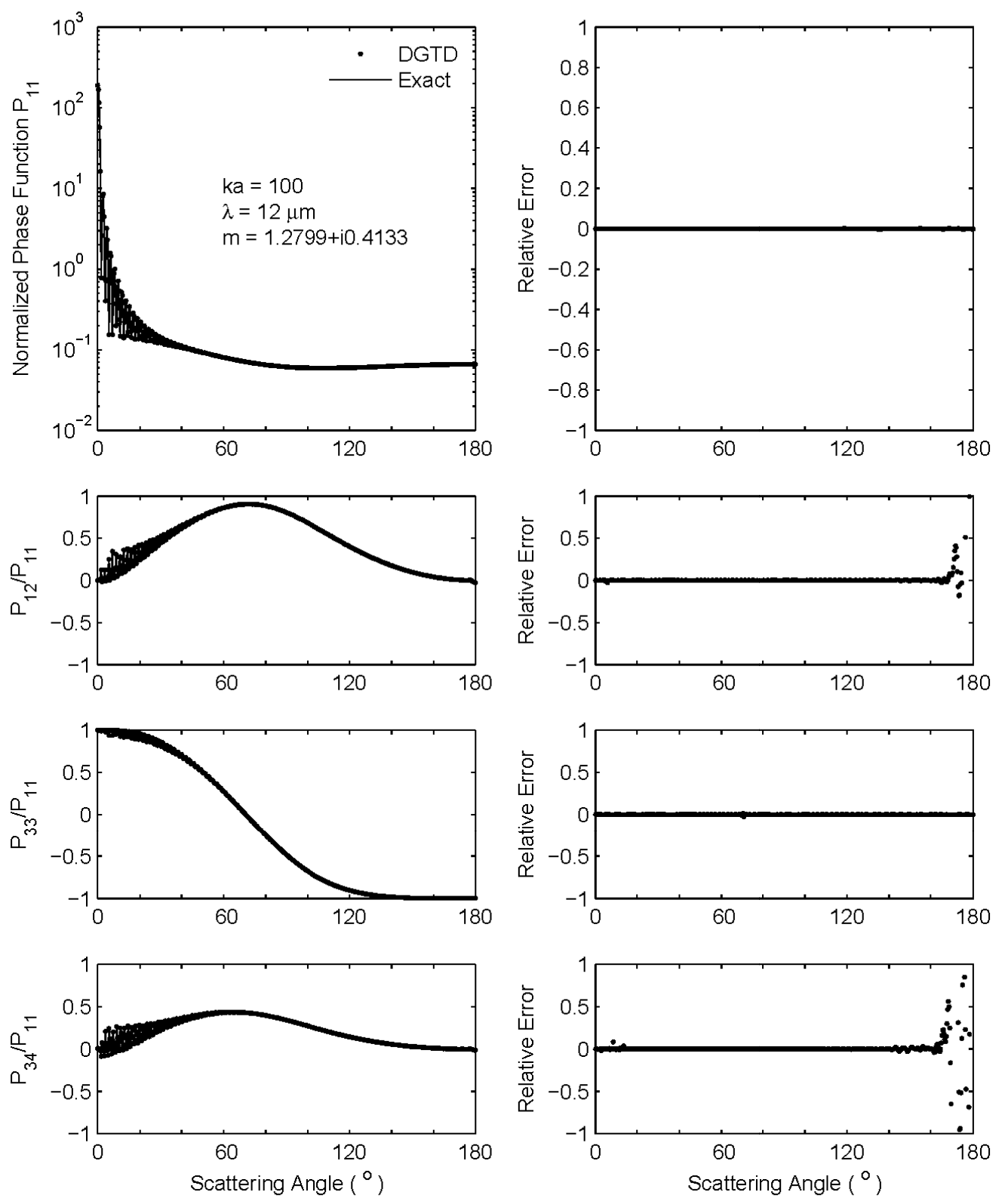

Fig. 6. The same as Fig. 5 except that the size parameter is 100 . 

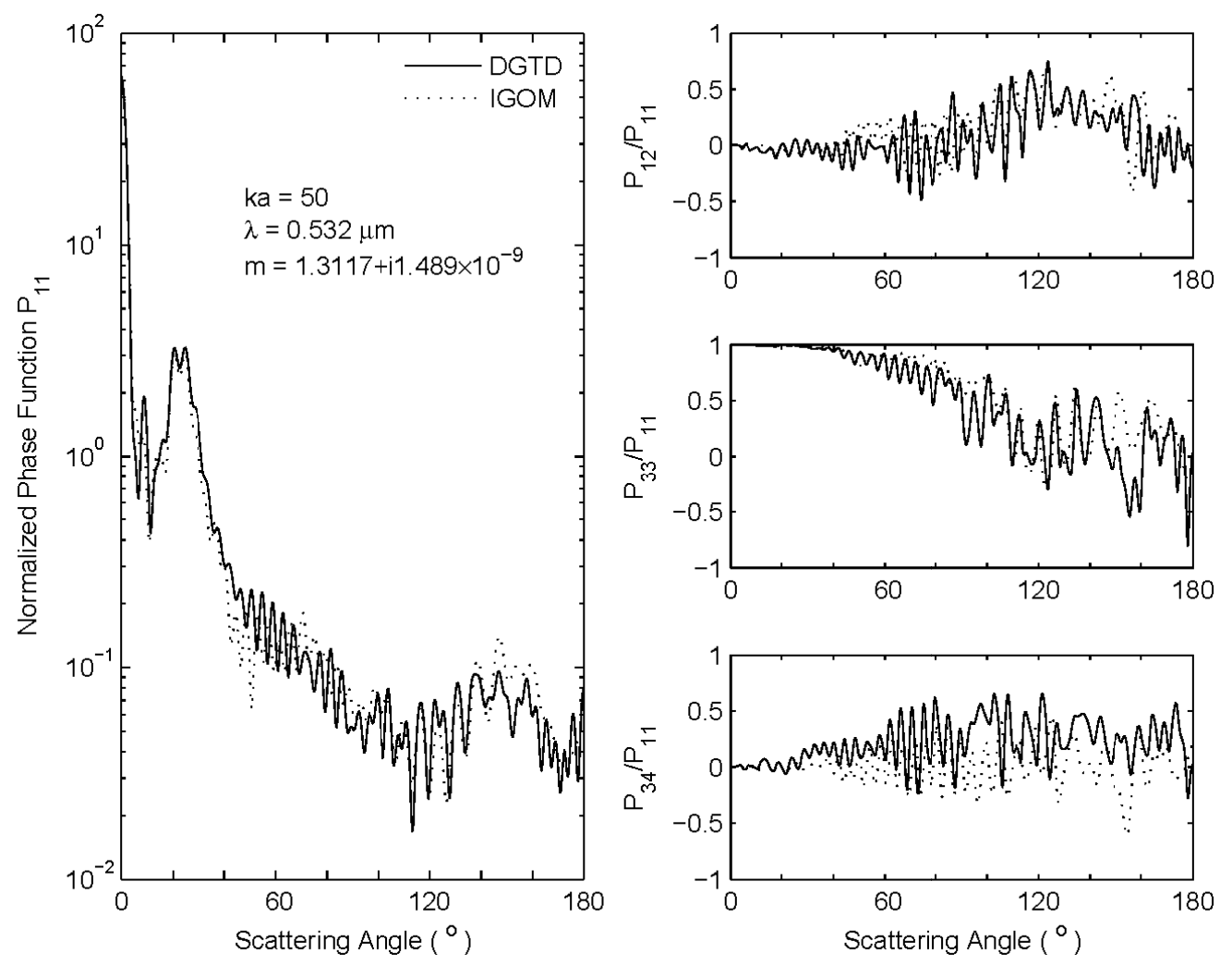

Fig. 7. Nonzero scattering phase matrix elements of randomly oriented 2-D hexagonal ice crystals with a size parameter of 50 and an incident wavelength of $0.532 \mu \mathrm{m}$. 

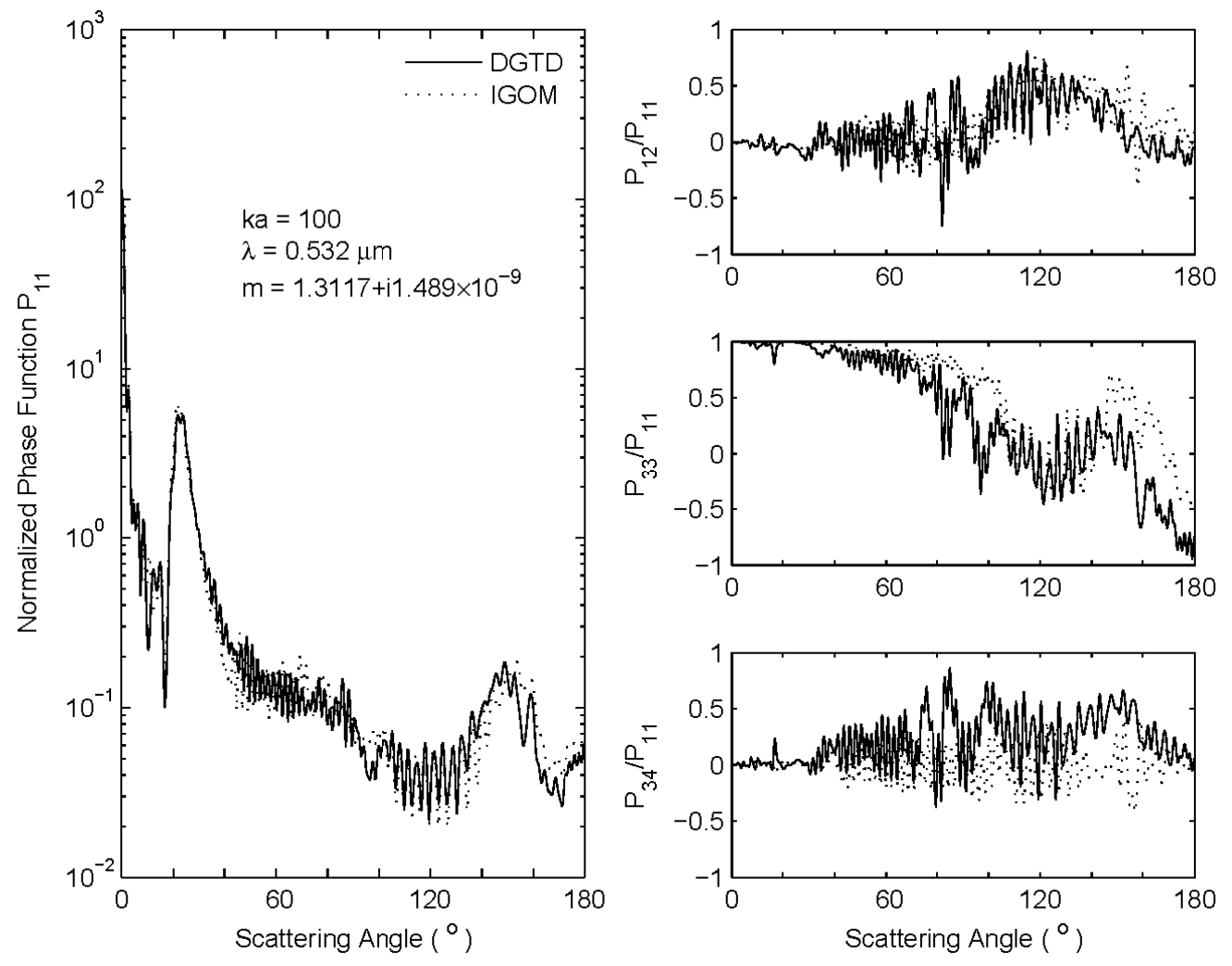

Fig. 8. The same as Fig. 6 except that the size parameter is 100 . 


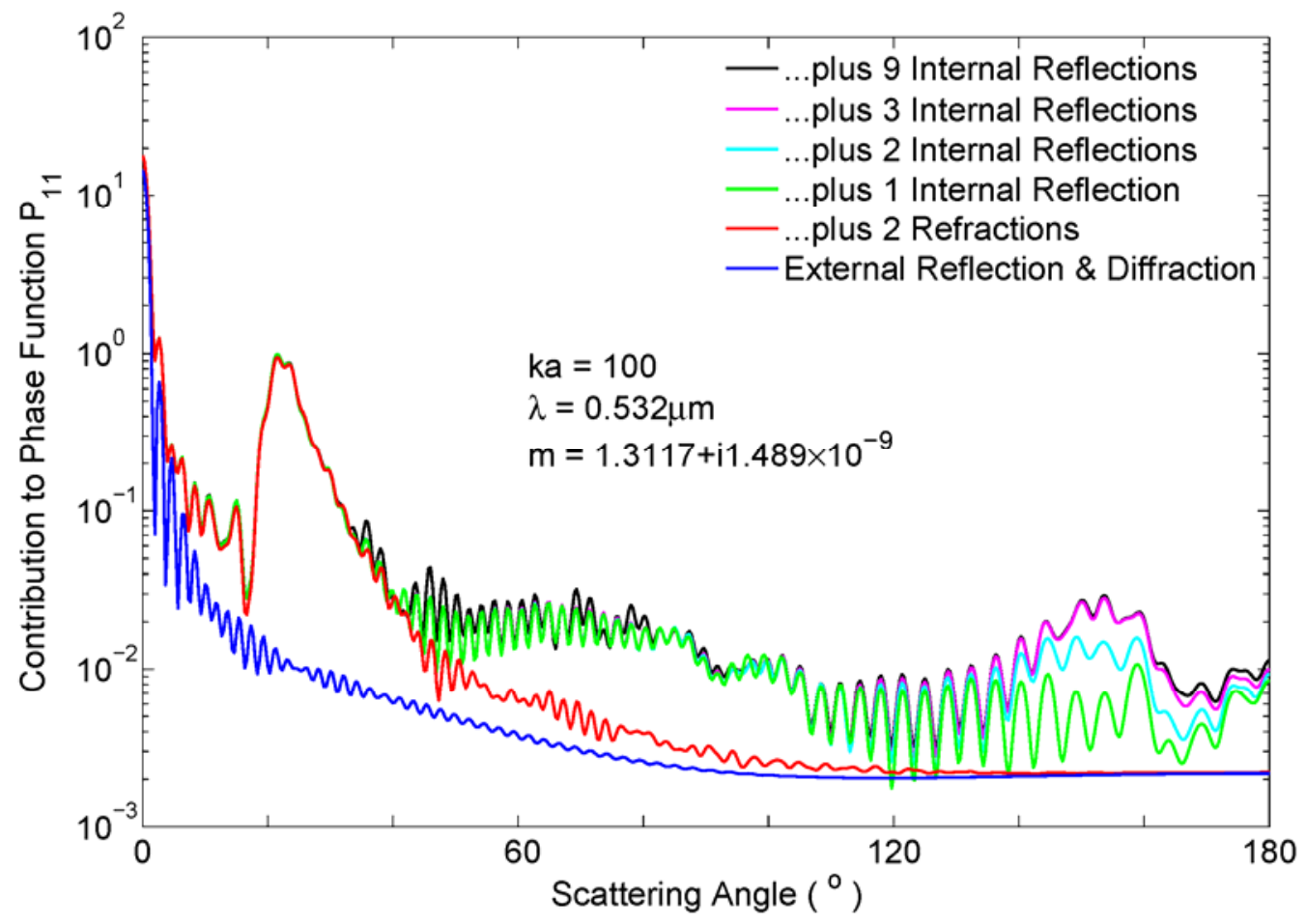

Fig. 9. Contributions of the rays of various orders to the phase function of 2D randomly oriented hexagonal ice crystals. 

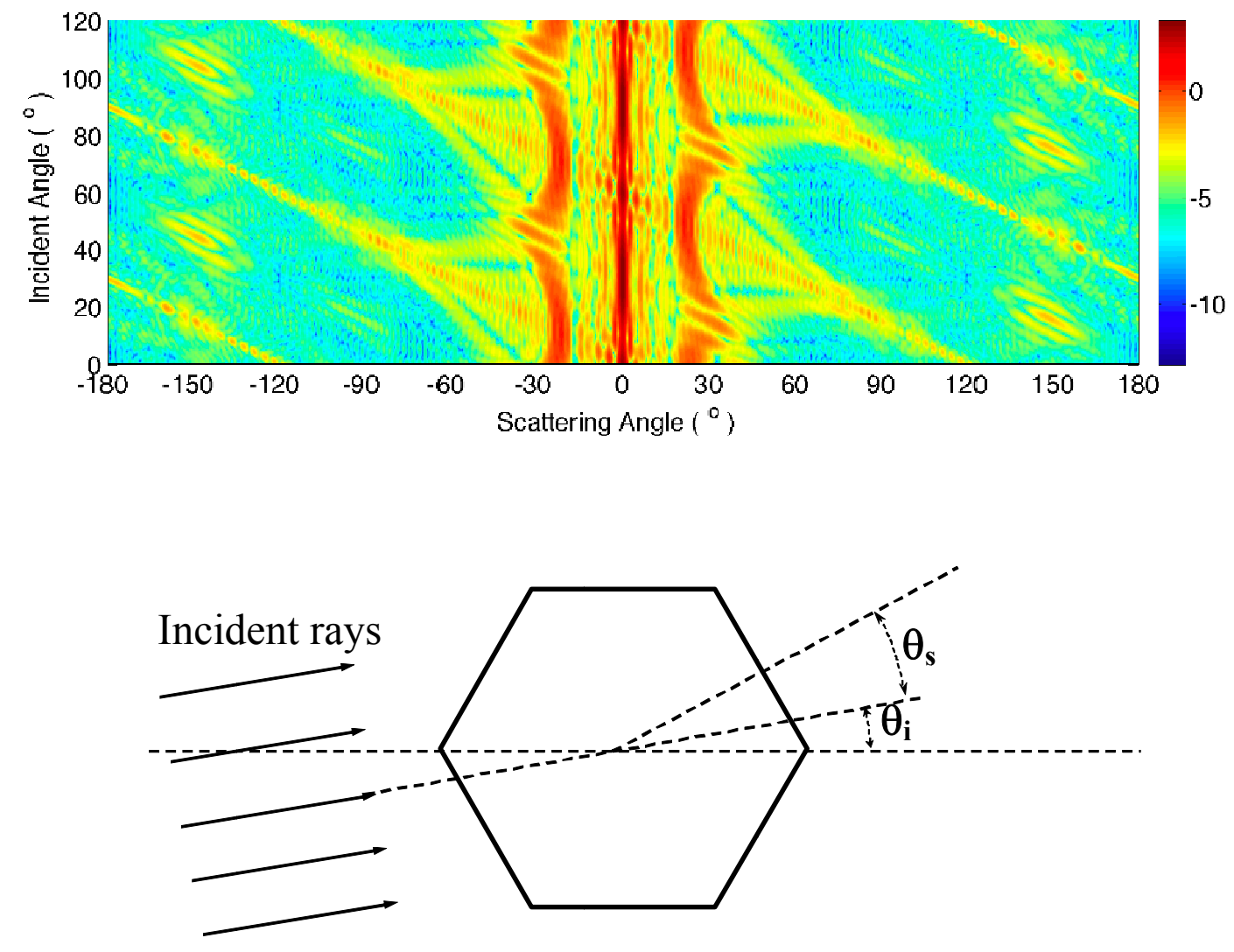

Fig. 10. Contour of the log of the phase function with respect to scattering angle and incident angle, for hexagonal particles with a size parameter of 100 and an incident wave length of $0.532 \mu \mathrm{m}$, where the incident angle $\left(\theta_{\mathrm{i}}\right)$ and scattering angle $\left(\theta_{\mathrm{i}}\right)$ are defined in the lower panel. 

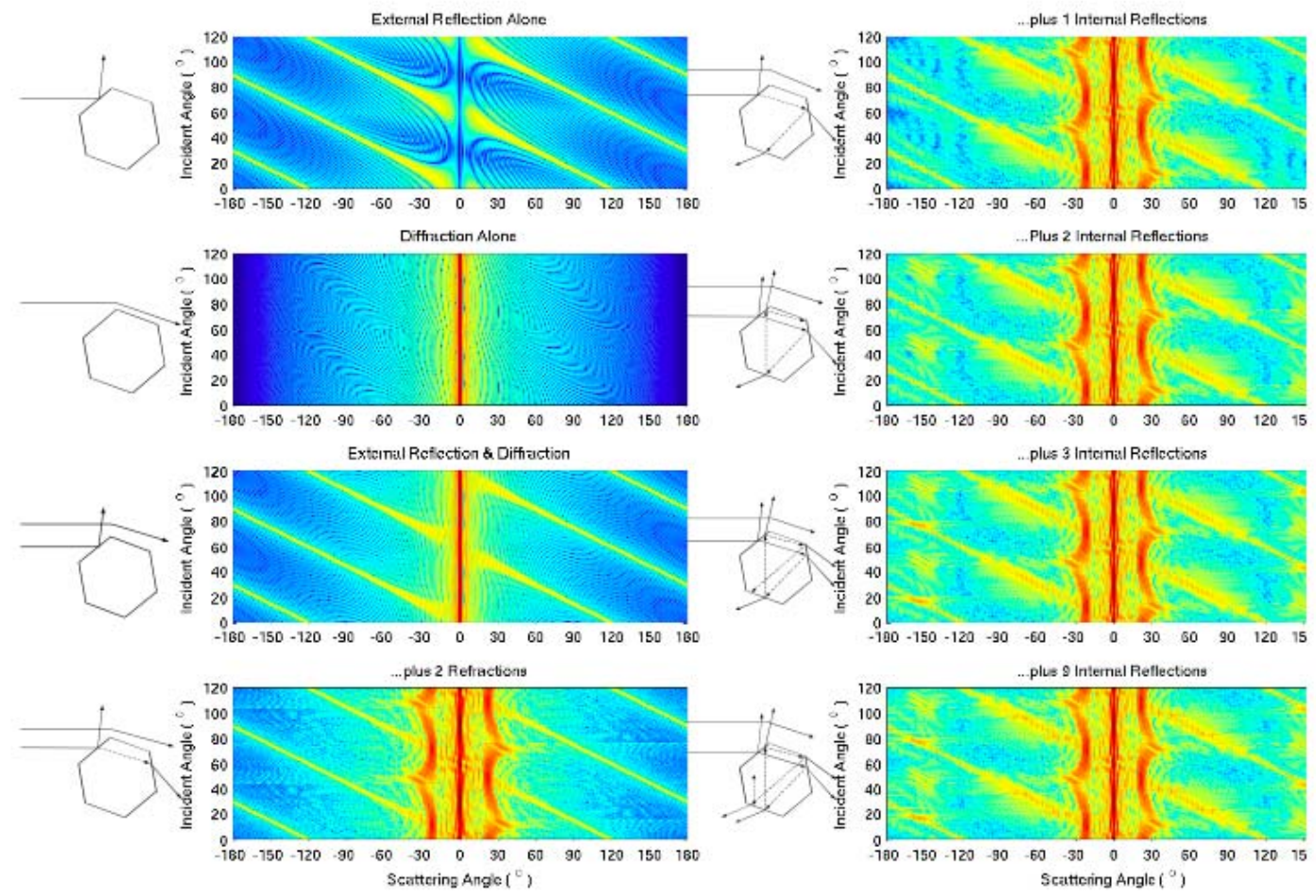

Fig. 11. Decomposition of the scattered field using geometrical optics. 


\section{3 materials}

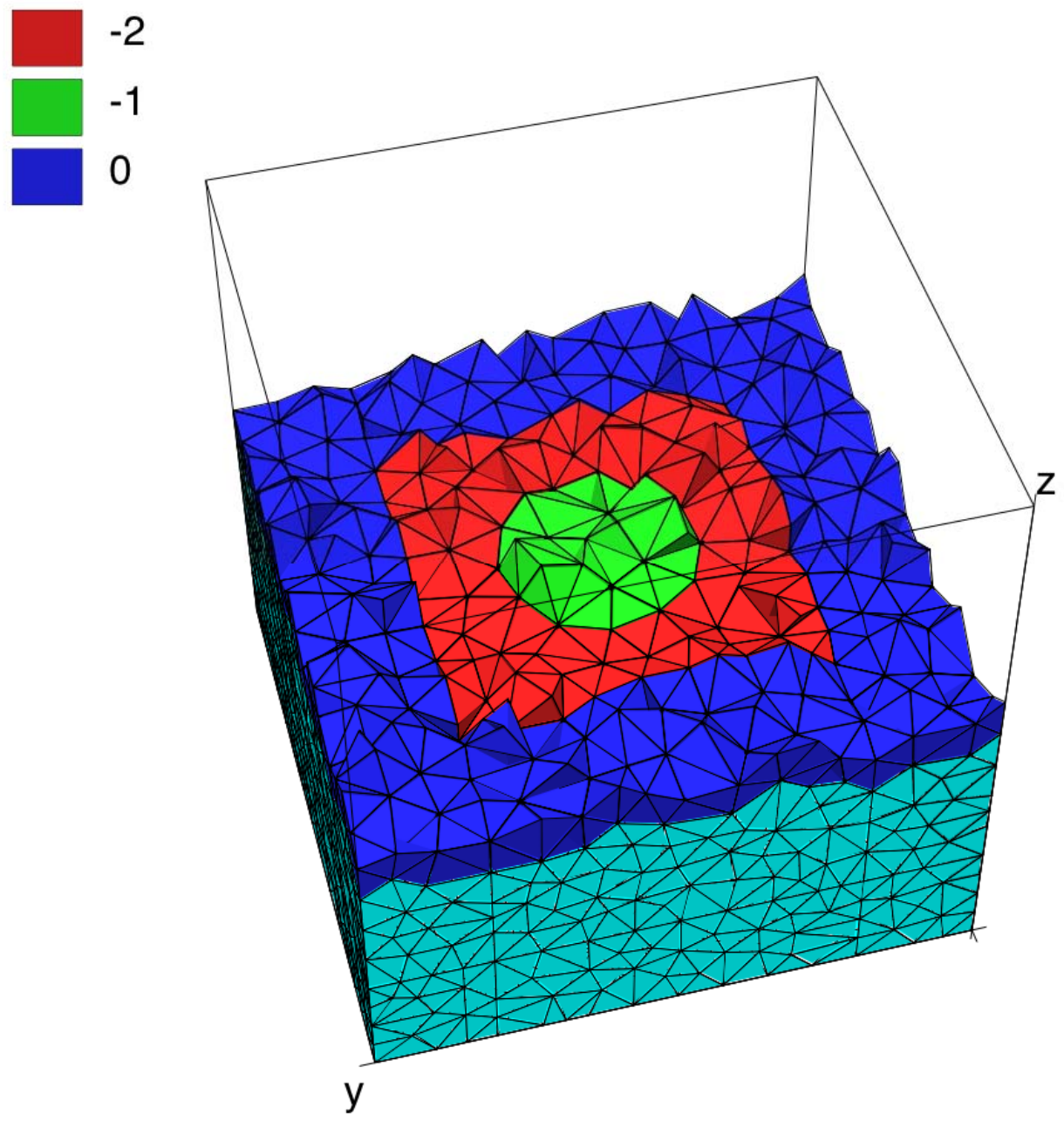

Fig. 12. An example of tetrahedron grid used in simulations of optical properties of a spherical particle. The green tetrahedrons make up the spherical particle, the red ones make up the "free space" and the blue ones make up the UPML. 


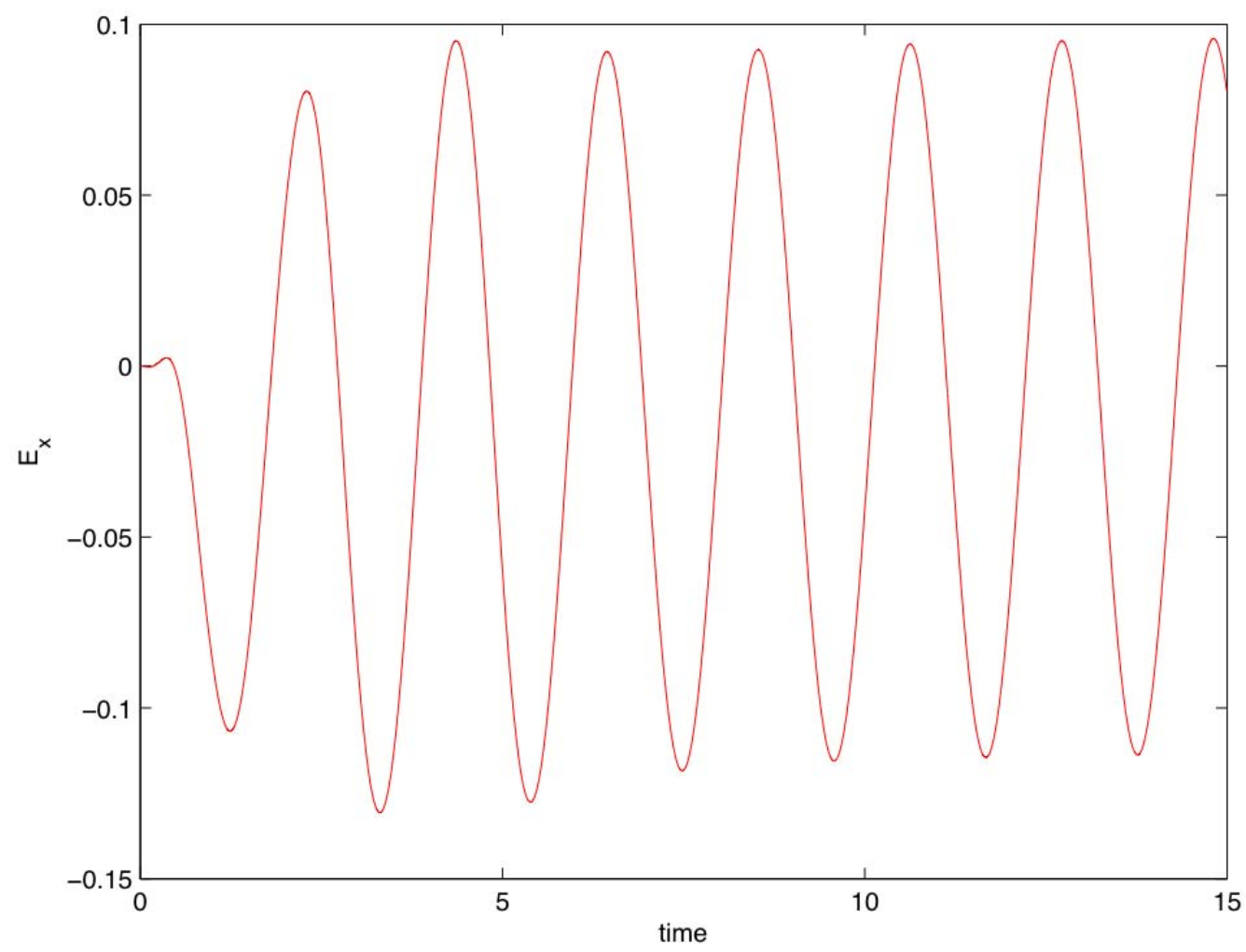

Fig. 13. The $\mathrm{x}$ component of the electric field at a point of the free space with respect to time. 
VITA

Name: $\quad$ Guanglin Tang

Address: $\quad$ Department of Atmospheric Sciences, MS 3150, Texas A\&M University, College Station, Texas 77843-3150 US

Email Address: tangguanglin@gmail.com

Education: $\quad$ B.S., Atmospheric Sciences, Peking University, 2008

M.S., Atmospheric Sciences, Texas A\&M University, 2010 